\title{
Construction of Malliavin differentiable strong solutions of SDEs under an integrability condition on the drift without the Yamada-Watanabe principle
}

\author{
David R. Baños ${ }^{\mathrm{a}}$, Sindre Duedahl ${ }^{\mathrm{a}}$, Thilo Meyer-Brandis ${ }^{\mathrm{b}}$ and Frank Proske ${ }^{\mathrm{a}}$ \\ ${ }^{a}$ University of Oslo, Moltke Moes vei 35 Niels Henrik Abels hus 0851 Oslo, Norway. \\ E-mail:davidru@math.uio.no; sindred@math.uio.no;proske@math.uio.no \\ ${ }^{\mathrm{b}}$ LMU University of Munich, Mathematics Institute, Theresienstr. 39, D-80333 Munich, Germany. E-mail: Meyer-Brandis@math.lmu.de
}

Received 13 January 2016; revised 5 April 2017; accepted 15 May 2017

\begin{abstract}
In this paper we aim at employing a compactness criterion of Da Prato, Malliavin, Nualart (C. R. Math. Acad. Sci. Paris 315 (1992) 1287-1291) for square integrable Brownian functionals to construct strong solutions of SDE's under an integrability condition on the drift coefficient. The obtained solutions turn out to be Malliavin differentiable and are used to derive a BismutElworthy-Li formula for solutions of the Kolmogorov equation. We emphasise that our approach exhibits high flexibility to study a variety of other types of stochastic (partial) differential equations as e.g. stochastic differential equations driven by fractional Brownian motion.
\end{abstract}

Résumé. Dans cet article, nous cherchons à utiliser un critère de compacité de Da Prato, Malliavin, Nualart pour les fonctionnelles browniennes de carré intégrable pour construire des solutions fortes d'EDS sous une condition d'intégrabilité sur le coefficient de dérive. Les solutions obtenues se révèlent être Malliavin-différentiables et sont utilisées pour dériver une formule BismutElworthy-Li pour des solutions de l'équation de Kolmogorov. Nous soulignons que notre approche présente une grande souplesse pour étudier une variété d'autres types d'équations différentielles stochastiques (aux dérivées partielles) comme par exemple des équations différentielles stochastiques conduites par un mouvement brownien fractionnaire.

MSC: 60H10; 60H07; 60H40; 60J60

Keywords: Strong solutions of SDEs; Malliavin calculus; Kolmogorov equation; Bismut-Elworthy-Li formula; Singular drift coefficient

\section{Introduction}

The object of study of this paper is the stochastic differential equation (SDE)

$$
X_{t}^{x}=x+\int_{0}^{t} b\left(s, X_{s}^{x}\right) d s+B_{t}, \quad 0 \leq t \leq T, x \in \mathbb{R}^{d},
$$

where $B$. is a $d$-dimensional Brownian motion on some complete probability space $(\Omega, \mathcal{F}, \mu)$ with respect to a $\mu$-completed Brownian filtration $\left\{\mathcal{F}_{t}\right\}_{0 \leq t \leq T}$ and where $b:[0, T] \times \mathbb{R}^{d} \rightarrow \mathbb{R}^{d}$ is a Borel-measurable function.

In this article we are interested in the analysis of strong solutions $X$. of the $\operatorname{SDE}(1)$, that is an $\left\{\mathcal{F}_{t}\right\}_{0 \leq t \leq T}$-adapted solution processes on $(\Omega, \mathcal{F}, \mu)$ when the drift coefficient is irregular, e.g. non-Lipschitzian or discontinuous.

A widely used construction method for strong solutions in this case in the literature is based on the so-called Yamada-Watanabe principle. Using this principle, a once constructed weak solution, that is a solution which is not 
necessarily a functional of the driving noise, combined with pathwise uniqueness gives a unique strong solution. So

Weak solution + Pathwise uniqueness $\Rightarrow \quad$ Unique strong solution.

Here, pathwise uniqueness means the following: If $X^{(1)}$ and $X^{(2)}$ are $\left\{\mathcal{F}_{t}^{(1)}\right\}_{0 \leq t \leq T^{-}}$and respectively $\left\{\mathcal{F}_{t}^{(2)}\right\}_{0 \leq t \leq T^{-}}$ adapted weak solutions on a probability space, then these solutions must coincide a.s. See [35]. In the milestone paper from 1974 [36], A.K. Zvonkin used the Yamada-Watanabe principle in the one-dimensional case in connection with PDE techniques to construct a unique strong solution to (1), when $b$ is merely bounded and measurable. Subsequently, the latter result was generalised by A.Y. Veretennikov [34] to the multidimensional case.

Important other and more recent results in this direction are e.g. by Krylov and Röckner [20] where the authors show existence and uniqueness using the Yamada-Watanabe principle in (2), see also the work by Gyöngy and Krylov [13], Krylov [19] and also the striking work [3] in the Hilbert space setting, where the authors use solutions of infinitedimensional Kolmogorov equations to obtain unique strong solutions of stochastic evolution equations with bounded and measurable drift for a.e. initial values.

In this article we want to employ a construction principle for strong solutions developed by Meyer-Brandis and Proske in [27]. This method which relies on a compactness criterion from Malliavin calculus for square integrable functionals of the Brownian motion [4] is used to establish strong existence of solutions of SDE's. Then the latter is combined with a principle (see Cherny [2] and the related works by Engelbert [6] and Jacod [16]) which is in diametrical opposition to the Yamada-Watanabe principle (2), to construct a unique strong solution of SDE's in the sense that

$$
\text { Strong existence }+ \text { Uniqueness in law } \Rightarrow \text { Strong uniqueness, }
$$

that is the existence of a strong solution to (1) and uniqueness in law of solutions imply the existence of a unique strong solution. A crucial consequence of our approach is the additional insight that the constructed solutions are regular in the sense of Malliavin differentiability.

We mention that this method has been recently applied in a series of other papers. See e.g. [25], where the authors obtain Malliavin differentiable solutions when the drift coefficient in $\mathbb{R}^{d}$ is bounded and measurable. Other applications pertain to the stochastic transport equation with singular coefficients $[28,29]$ or stochastic evolution equations in Hilbert spaces with bounded Hölder-continuous drift [12]. See also [14] in the case of a truncated $\alpha$-stable process as a driving noise and [1], where the authors study strong solutions of the SDE

$$
d X_{t}^{x}=b\left(t, X_{t}^{x}\right) d t+d B_{t}^{H}, \quad 0 \leq t \leq T, X_{0}^{x}=x \in \mathbb{R}^{d},
$$

for a singular drift coefficient $b \in L^{\infty}\left([0, T], L^{1}\left(\mathbb{R}^{d}\right) \cap L^{\infty}\left(\mathbb{R}^{d}\right)\right)$ and a non-Markovian driving noise given by a $d$-dimensional fractional Brownian motion $B_{t}^{H}$ with a Hurst parameter $H<1 / 2$.

Using the above mentioned new approach, one of the objectives of this paper is to construct Malliavin differentiable unique strong solutions to (1) under the integrability condition

$$
b \in L^{q}\left([0, T], L^{p}\left(\mathbb{R}^{d}, \mathbb{R}^{d}\right)\right)
$$

for $p \geq 2, q>2$ such that

$$
\frac{d}{p}+\frac{2}{q}<1
$$

The idea for the proof rests on a mixture of techniques in [25] and [9]. More precisely, we approximate in the first step the drift coefficient $b$ by smooth functions $b_{n}$ with compact support and apply the Itô-Tanaka-Zvonkin "trick" by transforming the solutions $X_{t}^{n, x}$ of (1) associated with the coefficients $b_{n}$ to processes

$$
Y_{t}^{n, x}:=X_{t}^{n, x}+U_{n}\left(t, X_{t}^{n, x}\right),
$$

where the processes $Y_{t}^{n, x}$ satisfy an equation with more regular coefficients than (1) given by

$$
d Y_{t}^{n, x}=\lambda U_{n}\left(t, X_{t}^{n, x}\right) d t+\left(\mathcal{I}_{d}+\nabla U_{n}\left(t, X_{t}^{n, x}\right)\right) d B_{t}
$$


for solutions $U_{n}$ to the backward PDE's

$$
\frac{\partial U_{n}}{\partial t}+\frac{1}{2} \Delta U_{n}+b_{n} \nabla U_{n}=\lambda U_{n}-b_{n}, \quad U_{n}(T, x)=0 .
$$

In the second step we use the compactness criterion for $L^{2}(\Omega)$ in [4] applied to the sequence $Y_{t}^{n, x}, n \geq 1$ in connection with Schauder-type of estimates of solutions of (4) and techniques from white noise analysis to show that

$$
Y_{t}^{n, x} \stackrel{n \rightarrow \infty}{\longrightarrow} Y_{t}^{x}
$$

in $L^{2}(\Omega)$ for all $t$ and that

$$
X_{t}^{x}=\varphi\left(t, Y_{t}^{x}\right),
$$

where $\varphi(t, \cdot)$ is the inverse of the function $x \mapsto x+U(t, x)$ for all $t$ and $U$ a solution of (4), is a Malliavin differentiable unique strong solution of (1).

We emphasise that we do not obtain new existence results for solutions of (1) under (3), but aim at demonstrating a new method based on Malliavin calculus for the construction of strong solutions of (1) under (3), which in addition grants the additional insight that such solutions must be Malliavin differentiable. So the novelty of our paper lies in the presentation of a new method for the construction of strong solutions to the SDE (1) and the regularity of such solutions in the sense of Malliavin differentiability as a consequence of this approach. In achieving the latter result, we employ Schauder-type of estimates of solutions of (4) and establish in this paper the following new key estimates of Malliavin derivatives $D$. of solutions (Theorem 2.6), which enable the application of the $L^{2}(\Omega)$-compactness criterion in [4]:

$$
\begin{aligned}
& \sup _{r \in[0, t]}\left\|D_{r} X_{t}\right\|_{L^{2}(\Omega)}^{2} \leq C\left(\|b\|_{L_{p}^{q}}\right), \\
& \left\|D_{r^{\prime}} X_{t}-D_{r} X_{t}\right\|_{L^{2}(\Omega)}^{2} \leq C\left(\|b\|_{L_{p}^{q}}\right)\left|r^{\prime}-r\right|^{\delta}
\end{aligned}
$$

for all $0 \leq r^{\prime} \leq r \leq t$ and some $\delta>0$, where $C$ is a positive continuous function on $[0, \infty), X$. is the unique strong solution to (1) associated with the vector field $b \in C_{0}^{\infty}\left((0, T) \times \mathbb{R}^{d}\right)$ and where

$$
\|b\|_{L_{p}^{q}}=\left(\int_{0}^{T}\left(\int_{\mathbb{R}^{d}}|b(t, x)|^{p} d x\right)^{q / p}\right)^{1 / q} .
$$

Using the Malliavin regularity of solutions under the integrability condition (3), we also derive a Bismut-ElworthyLi formula for the representation of first order derivatives of solutions of Kolmogorov equations.

Our paper is organised as follows: In Section 2 we present our main results on the construction of strong solutions (Theorem 2.1 and Theorem 2.15).

As an application of the results obtained in Section 2 we establish in Section 3 a Bismut-Elworthy-Li type of formula with respect to solutions of Kolmogorov equations.

\section{Main results}

In this section, we want to further develop the ideas introduced in [9] and [27] to derive Malliavin differentiable strong solutions of stochastic differential equations with irregular coefficients. More precisely, we aim at analysing the SDE's of the form

$$
d X_{t}=b\left(t, X_{t}\right) d t+d B_{t}, \quad 0 \leq t \leq T, X_{0}=x \in \mathbb{R}^{d},
$$

where the drift coefficient $b:[0, T] \times \mathbb{R}^{d} \longrightarrow \mathbb{R}^{d}$ is a Borel measurable function satisfying some integrability condition and $B$. is a $d$-dimensional Brownian motion with respect to the stochastic basis

$$
(\Omega, \mathcal{F}, \mu),\left\{\mathcal{F}_{t}\right\}_{0 \leq t \leq T}
$$


for the $\mu$-augmented filtration $\left\{\mathcal{F}_{t}\right\}_{0 \leq t \leq T}$ generated by $B$. At the end of this section we shall also apply our technique to equations with more general diffusion coefficients (Theorem 2.15).

Consider the space

$$
L_{p}^{q}:=L^{q}\left([0, T], L^{p}\left(\mathbb{R}^{d}, \mathbb{R}^{d}\right)\right)
$$

for $p, q \in \mathbb{R}$ satisfying the following condition

$$
p>2, \quad q>2 \text { and } \frac{d}{p}+\frac{2}{q}<1
$$

and denote by $|\cdot|$ the Euclidean norm in $\mathbb{R}^{d}$. The Banach space $L_{p}^{q}$ is endowed with the norm

$$
\|f\|_{L_{p}^{q}}=\left(\int_{0}^{T}\left(\int_{\mathbb{R}^{d}}|f(t, x)|^{p} d x\right)^{q / p} d t\right)^{1 / q}<\infty
$$

for $f \in L_{p}^{q}$.

The main goal of the paper is to show that SDE's of the type (5) with drift coefficient $b$ satisfying the integrability condition given in (8) admit strong solutions that are unique and in addition, Malliavin differentiable. As mentioned in the Introduction, the existence is not new, it was proven by Krylov and Röckner in [20] using a method based on PDE theory in conjunction with the Yamada-Watanabe principle. Here, we employ a new method which directly provides Malliavin differentiable strong solutions, then weak uniqueness is enough to ensure pathwise uniqueness, see [2].

So, our main result is the following theorem:

Theorem 2.1. Suppose that the drift coefficient $b:[0, T] \times \mathbb{R}^{d} \rightarrow \mathbb{R}^{d}$ in (5) belongs to $L_{p}^{q}$. Then there exists a unique global strong solution $X$ to equation (5) such that $X_{t}$ is Malliavin differentiable for all $0 \leq t \leq T$.

An important step of the proof of Theorem 2.1 is directly based on the study of the regularity of solutions to the following PDE, associated to equation SDE (5).

$$
\partial_{t} U(t, x)+b(t, x) \cdot \nabla U(t, x)+\frac{1}{2} \Delta U(t, x)-\lambda U(t, x)+b=0, \quad t \in[0, T], U(T, x)=0,
$$

where $U:[0, T] \times \mathbb{R}^{d} \rightarrow \mathbb{R}^{d}, \lambda>0$ and $b \in L_{p}^{q}$. We will make use of the following family of vector fields $\gamma_{t}: \mathbb{R}^{d} \rightarrow$ $\mathbb{R}^{d}$ defined by $\gamma_{t}(x)=x+U(t, x), t \in[0, T]$ that are introduced in [11]. In [8] they are proven to define a family of $C^{1}$-diffeomorphisms in our context.

The following result is due to [8] and establishes the well-posedness of the above PDE problem in a certain space.

First, recall the definition of the following functional spaces

$$
\mathbb{H}_{\alpha, p}^{q}=L^{q}\left([0, T], W^{\alpha, p}\left(\mathbb{R}^{d}\right)\right), \quad \mathbb{H}_{p}^{\beta, q}=W^{\beta, q}\left([0, T], L^{p}\left(\mathbb{R}^{d}\right)\right)
$$

and

$$
H_{\alpha, p}^{q}=\mathbb{H}_{\alpha, p}^{q} \cap \mathbb{H}_{p}^{1, q} .
$$

The norm in $H_{\alpha, p}^{q}$ can be taken to be

$$
\|U\|_{H_{\alpha, p}^{q}} \equiv\|U\|_{\mathbb{H}_{\alpha, p}^{q}}+\left\|\partial_{t} U\right\|_{L_{p}^{q}}
$$

Theorem 2.2. Let $p, q$ be such that $p \geq 2, q>2$ and $\frac{d}{p}+\frac{2}{q}<1$ and $\lambda>0$. Consider two vector fields $b, \Phi \in L_{p}^{q}$. Then there exists a unique solution of the backward parabolic system

$$
\partial_{t} U+\frac{1}{2} \Delta U+b \cdot \nabla U-\lambda U+\Phi=0, \quad t \in[0, T], u(T, x)=0
$$


belonging to the space

$$
H_{2, p}^{q}:=L^{q}\left([0, T], W^{2, p}\left(\mathbb{R}^{d}\right)\right) \cap W^{1, q}\left([0, T], L^{p}\left(\mathbb{R}^{d}\right)\right),
$$

i.e. there exists a constant $C>0$ depending only on $d, p, q, T, \lambda$ and $\|b\|_{L_{p}^{q}}$ such that

$$
\|U\|_{H_{2, p}^{q}} \leq C\|\Phi\|_{L_{p}^{q}}
$$

The following result is a part of [20, Lemma 10.2] that gives us some properties on the regularity of $U \in H_{2, p}^{q}$ that we will need for the proof of Theorem 2.1.

Lemma 2.3. Let $p, q \in(1, \infty)$ such that $\frac{d}{p}+\frac{2}{q}<1$ and $U \in H_{2, p}^{q}$, then $\nabla U$ is Hölder continuous in $(t, x) \in[0, T] \times$ $\mathbb{R}^{d}$, namely for any $\varepsilon \in(0,1)$ satisfying

$$
\varepsilon+\frac{d}{p}+\frac{2}{q}<1
$$

there exists a constant $C>0$ depending only on $p, q$ and $\varepsilon$ such that for all $s, t \in[0, T]$ and $x, y \in \mathbb{R}^{d}, x \neq y$

$$
\begin{aligned}
& \|\nabla U(t, x)-\nabla U(s, x)\| \leq C|t-s|^{\varepsilon / 2}\|\nabla U\|_{H_{2, p}^{q}}^{1-1 / q-\varepsilon / 2}\left\|\partial_{t} U\right\|_{L_{p}^{q}}^{1 / q+\varepsilon / 2}, \\
& \|\nabla U(t, x)\|+\frac{\|\nabla U(t, x)-\nabla U(t, y)\|}{|x-y|^{\varepsilon}} \leq C T^{-1 / q}\left(\|U\|_{H_{2, p}^{q}}+T\left\|\partial_{t} U\right\|_{L_{p}^{q}}\right),
\end{aligned}
$$

where $\|\cdot\|$ denotes any norm in $\mathbb{R}^{d \times d}$.

Our method to directly construct strong solutions is actually motivated by the following observation in [22] and [26] (see also [27]).

Proposition 2.4. Suppose that the drift coefficient $b:[0, T] \times \mathbb{R}^{d} \longrightarrow \mathbb{R}^{d}$ in (5) is bounded and Lipschitz continuous. Then the unique strong solution $X_{t}=\left(X_{t}^{1}, \ldots, X_{t}^{d}\right)$ of (5) has the explicit representation

$$
\varphi\left(t, X_{t}^{i}(\omega)\right)=E_{\widetilde{\mu}}\left[\varphi\left(t, \widetilde{B}_{t}^{i}(\widetilde{\omega})\right) \mathcal{E}_{T}^{\diamond}(b)\right]
$$

for all $\varphi:[0, T] \times \mathbb{R} \longrightarrow \mathbb{R}$ such that $\varphi\left(t, B_{t}^{i}\right) \in L^{2}(\Omega)$ for all $0 \leq t \leq T, i=1, \ldots, d$. The random element $\mathcal{E}_{T}^{\diamond}(b)$ is given by

$$
\begin{aligned}
\mathcal{E}_{T}^{\diamond}(b)(\omega, \widetilde{\omega}):= & \exp ^{\diamond}\left(\sum_{j=1}^{d} \int_{0}^{T}\left(W_{s}^{j}(\omega)+b^{j}\left(s, \widetilde{B}_{s}(\widetilde{\omega})\right)\right) d \widetilde{B}_{s}^{j}(\widetilde{\omega})\right. \\
& \left.-\frac{1}{2} \int_{0}^{T}\left(W_{s}^{j}(\omega)+b^{j}\left(s, \widetilde{B}_{s}(\widetilde{\omega})\right)\right)^{\diamond 2} d s\right) .
\end{aligned}
$$

Here $(\widetilde{\Omega}, \widetilde{\mathcal{F}}, \widetilde{\mu}),\left(\widetilde{B}_{t}\right)_{t \geq 0}$ is a copy of the quadruple $(\Omega, \mathcal{F}, \mu),\left(B_{t}\right)_{t \geq 0}$ in $(6)$. Further $E_{\widetilde{\mu}}$ denotes a Pettis integral of random elements $\Phi: \widetilde{\widetilde{\Omega}} \longrightarrow(\mathcal{S})^{*}$ with respect to the measure $\tilde{\mu}$. The Wick product $\diamond$ in the Wick exponential of (15) (see (60)) is taken with respect to $\mu$ and $W_{t}^{j}$ is the white noise of $B_{t}^{j}$ in the Hida space $(\mathcal{S})^{*}$ (see (57)). The stochastic integrals $\int_{0}^{T} \phi(t, \widetilde{\omega}) d \widetilde{B}_{S}^{j}(\widetilde{\omega})$ in $(15)$ are defined for predictable integrands $\phi$ with values in the conuclear space $(\mathcal{S})^{*}$. See e.g. [17] for definitions. The other integral type in (15) is to be understood in the sense of Pettis. 
Remark 2.5. Let $0=t_{1}^{n}<t_{2}^{n}<\cdots<t_{m_{n}}^{n}=T$ be a sequence of partitions of the interval [0,T] with $\max _{i=1}^{m_{n}-1} \mid t_{i+1}^{n}-$ $t_{i}^{n} \mid \longrightarrow 0$. Then the stochastic integral of the white noise $W^{j}$ can be approximated as follows:

$$
\int_{0}^{T} W_{s}^{j}(\omega) d \widetilde{B}_{s}^{j}(\widetilde{\omega})=\lim _{n \longrightarrow \infty} \sum_{i=1}^{m_{n}}\left(\widetilde{B}_{t_{i+1}^{n}}^{j}(\widetilde{\omega})-\widetilde{B}_{t_{i}^{n}}^{j}(\widetilde{\omega})\right) W_{t_{i}^{n}}^{j}(\omega)
$$

in $L^{2}\left(\lambda \times \tilde{\mu} ;(\mathcal{S})^{*}\right)$. For more information about stochastic integration on conuclear spaces the reader is referred to $[17]$.

In the sequel we shall use the notation $Y_{t}^{i, b}$ for the expectation on the right hand side of (14) for $\varphi(t, x)=x$, that is

$$
Y_{t}^{i, b}:=E_{\widetilde{\mu}}\left[\widetilde{B}_{t}^{(i)} \mathcal{E}_{T}^{\diamond}(b)\right]
$$

for $i=1, \ldots, d$. We set

$$
Y_{t}^{b}=\left(Y_{t}^{1, b}, \ldots, Y_{t}^{d, b}\right)
$$

The form of Formula (14) in Proposition 2.4 actually gives rise to the conjecture that the expectation on the right hand side of $Y_{t}^{b}$ in (16) may also define solutions of (5) for drift coefficients $b$ lying in $L_{p}^{q}$.

Our method to construct strong solutions of equation (5) which are Malliavin differentiable is essentially based on four steps.

- First, we consider a sequence of compactly supported smooth functions $b_{n}:[0, T] \times \mathbb{R}^{d} \rightarrow \mathbb{R}^{d}, n \geq 0$ such that $\sup _{n \geq 0}\left\|b_{n}\right\|_{L_{p}^{q}}<\infty$ approximating $b \in L_{p}^{q}$ a.e. with respect to the Lebesgue measure and then we prove that the sequence of strong solutions $X_{t}^{n}=Y_{t}^{b_{n}}, n \geq 1$, is relatively compact in $L^{2}\left(\Omega ; \mathbb{R}^{d}\right)$ (Corollary 2.9) for every $t \in[0, T]$. The main tool to verify compactness is the bound in Theorem 2.6 in connection with a compactness criterion in terms of Malliavin derivatives obtained in [4] (see Appendix B). This step is one of the main contributions of this paper.

- Secondly, given a merely measurable drift coefficient $b$ in the space $L_{p}^{q}$, we show that $Y_{t}^{b}, t \in[0, T]$ is a generalized process in the Hida distribution space and we invoke the $S$-transform (58) to prove that for a given sequence of a.e. approximating, smooth coefficients $b_{n}$ with compact support such that $\sup _{n \geq 0}\left\|b_{n}\right\|_{L_{p}^{q}}$, a subsequence of the corresponding strong solutions $X_{t}^{n_{j}}=Y_{t}^{b_{n_{j}}}$ fulfils

$$
Y_{t}^{b_{n_{j}}} \rightarrow Y_{t}^{b}
$$

as $j \rightarrow \infty$ in $L^{2}\left(\Omega ; \mathbb{R}^{d}\right)$ for $0 \leq t \leq T$ (Lemma 2.12). In fact, the convergence holds for the whole sequence.

- Using a certain transformation property for $Y_{t}^{b}$ (Lemma 2.14) we directly show that $Y_{t}^{b}$ is a Malliavin differentiable solution to (5).

- Finally, using the argument by Cherny, see [2] (see also Engelbert [6]), as outlined in the Introduction, we only need to verify uniqueness in law in order to guarantee pathwise unique solutions.

We turn now to the first step of our procedure. The successful completion of the first step relies on the following essential theorem which provides new types of Malliavin bounds of solutions to (1) in terms of the $L_{p}^{q}$-norm:

Theorem 2.6. Let $b_{n}:[0, T] \times \mathbb{R}^{d} \rightarrow \mathbb{R}^{d}, n \geq 1$ be a sequence of functions in $\mathcal{C}_{0}^{\infty}\left([0, T] \times \mathbb{R}^{d}\right)$ (space of infinitely often differentiable functions with compact support) approximating $b \in L_{p}^{q}$ a.e. such that $\sup _{n \geq 0}\left\|b_{n}\right\|_{L_{p}^{q}}<\infty$. Denote by $X_{t}^{n, x}$ the strong solution of SDE (5) with drift coefficient $b_{n}$ for each $n \geq 0$. Then for every $t \in[0, T], 0 \leq r^{\prime} \leq r \leq t$ there exist a $0<\delta<1$ and a function $C: \mathbb{R} \rightarrow[0, \infty)$ depending only on $p, q, d, \delta$ and $T$ such that

$$
E\left[\left\|D_{r^{\prime}} X_{t}^{n, x}-D_{r} X_{t}^{n, x}\right\|^{2}\right] \leq C\left(\left\|b_{n}\right\|_{L_{p}^{q}}\right)\left|r^{\prime}-r\right|^{\delta}
$$


with

$$
\sup _{n \geq 1} C\left(\left\|b_{n}\right\|_{L_{p}^{q}}\right)<\infty
$$

Here $\|\cdot\|$ denotes any norm in $\mathbb{R}^{d \times d}$.

\section{Moreover,}

$$
\sup _{n \geq 1} \sup _{r \in[0, T]} E\left[\left\|D_{r} X_{t}^{n, x}\right\|^{p}\right]<\infty
$$

for all $p \geq 2$.

Proof. Throughout the proof we will denote by $C_{*}: \mathbb{R} \rightarrow[0, \infty)$ any function depending on the parameters $*$. We will also use the symbol $\lesssim$ to denote less or equal up to a positive real constant independent of $n$.

We will prove the above estimates by considering the solution of the associated PDE presented in (9) with $b_{n}, n \geq 0$ in place of $b$ which we denote by $U_{n}, n \geq 0$ and then using the results introduced at the beginning of this section on the regularity of its solution.

First, let us introduce a new process that will be useful for this purpose. Consider for each $n \geq 0$ and $t \in[0, T]$ the functions $\gamma_{t, n}: \mathbb{R}^{d} \rightarrow \mathbb{R}^{d}$ defined as $\gamma_{t, n}(x)=x+U_{n}(t, x)$. It turns out, see [8, Lemma 3.5], that the functions $\gamma_{t, n}, t \in[0, T], n \geq 0$ define a family of $C^{1}$-diffeomorphisms on $\mathbb{R}^{d}$. Furthermore, consider the auxiliary process $\tilde{X}_{t}^{n, x}:=\gamma_{t, n}\left(X_{t}^{n, x}\right), t \in[0, T], n \geq 1$. One checks using Itô's formula and (9) that $\tilde{X}_{t}^{n, x}$ satisfies the following SDE

$$
d \tilde{X}_{t}^{n, x}=\lambda U_{n}\left(t, \gamma_{t, n}^{-1}\left(\tilde{X}_{t}^{n, x}\right)\right) d t+\left(\mathcal{I}_{d}+\nabla U_{n}\left(t, \gamma_{t, n}^{-1}\left(\tilde{X}_{t}^{n, x}\right)\right)\right) d B_{t}, \quad \tilde{X}_{0}^{n, x}=x+U_{n}(0, x)
$$

which is equivalent to $\operatorname{SDE}(5)$ if we replace $b$ by $b_{n}, n \geq 1$. Using the chain rule for Malliavin derivatives (see e.g. [30]) we see that for $0 \leq r \leq t$,

$$
D_{r} \tilde{X}_{t}^{n, x}=\nabla \gamma_{t, n}\left(X_{t}^{n, x}\right) D_{r} X_{t}^{n, x} .
$$

Because of Lemma B.4 it suffices to prove the estimates (17) and (18) for the process $\tilde{X}_{t}^{n, x}$.

Since $b_{n}$ are now smooth we have that (19) admits a unique strong solution which takes the form

$$
\tilde{X}_{t}^{n, x}=x+U_{n}(0, x)+\lambda \int_{0}^{t} U_{n}\left(s, \gamma_{s, n}^{-1}\left(\tilde{X}_{s}^{n, x}\right)\right) d s+\int_{0}^{t}\left(\mathcal{I}_{d}+\nabla U_{n}\left(s, \gamma_{s, n}^{-1}\left(\tilde{X}_{s}^{n, x}\right)\right)\right) d B_{s} .
$$

Then the Malliavin derivative of $\tilde{X}_{t}^{n, x}$ for $0 \leq r \leq t$, which exists (see e.g. [30]), is

$$
\begin{aligned}
D_{r} \tilde{X}_{t}^{n, x}= & \mathcal{I}_{d}+\nabla U_{n}\left(r, \gamma_{r, n}^{-1}\left(\tilde{X}_{r}^{n, x}\right)\right) \\
& +\lambda \int_{r}^{t} \nabla U_{n}\left(s, \gamma_{s, n}^{-1}\left(\tilde{X}_{s}^{n, x}\right)\right) \nabla \gamma_{s, n}^{-1}\left(\tilde{X}_{s}^{n, x}\right) D_{r} \tilde{X}_{s}^{n, x} d s \\
& +\int_{r}^{t} \nabla^{2} U_{n}\left(s, \gamma_{s, n}^{-1}\left(\tilde{X}_{s}^{n, x}\right)\right) \nabla \gamma_{s, n}^{-1}\left(\tilde{X}_{s}^{n, x}\right) D_{r} \tilde{X}_{s}^{n, x} d B_{s} .
\end{aligned}
$$

Denote for simplicity, $Z_{r, t}^{n}:=D_{r} \tilde{X}_{t}^{n, x}$. Then for $r^{\prime}<r$ we can write

$$
\begin{aligned}
Z_{r^{\prime}, t}^{n}-Z_{r, t}^{n}= & \nabla U_{n}\left(r^{\prime}, \gamma_{r^{\prime}, n}^{-1}\left(\tilde{X}_{r^{\prime}}^{n, x}\right)\right)-\nabla U_{n}\left(r, \gamma_{r, n}^{-1}\left(\tilde{X}_{r}^{n, x}\right)\right) \\
& +\lambda \int_{r^{\prime}}^{r} \nabla U_{n}\left(s, \gamma_{s, n}^{-1}\left(\tilde{X}_{s}^{n, x}\right)\right) \nabla \gamma_{s, n}^{-1}\left(\tilde{X}_{s}^{n, x}\right) Z_{r^{\prime}, s}^{n} d s \\
& +\lambda \int_{r}^{t} \nabla U_{n}\left(s, \gamma_{s, n}^{-1}\left(\tilde{X}_{s}^{n, x}\right)\right) \nabla \gamma_{s, n}^{-1}\left(\tilde{X}_{s}^{n, x}\right)\left(Z_{r^{\prime}, s}^{n}-Z_{r, s}^{n}\right) d s
\end{aligned}
$$




$$
\begin{aligned}
& \quad+\int_{r^{\prime}}^{r} \nabla^{2} U_{n}\left(s, \gamma_{s, n}^{-1}\left(\tilde{X}_{s}^{n, x}\right)\right) \nabla \gamma_{s, n}^{-1}\left(\tilde{X}_{s}^{n, x}\right) Z_{r^{\prime}, s}^{n} d B_{s} \\
& +\int_{r}^{t} \nabla^{2} U_{n}\left(s, \gamma_{s, n}^{-1}\left(\tilde{X}_{s}^{n, x}\right)\right) \nabla \gamma_{s, n}^{-1}\left(\tilde{X}_{s}^{n, x}\right)\left(Z_{r^{\prime}, s}^{n}-Z_{r, s}^{n}\right) d B_{s} \\
& =Z_{r^{\prime}, r}^{n}-Z_{r, r}^{n} \\
& +\lambda \int_{r}^{t} \nabla U_{n}\left(s, \gamma_{s, n}^{-1}\left(\tilde{X}_{s}^{n, x}\right)\right) \nabla \gamma_{s, n}^{-1}\left(\tilde{X}_{s}^{n, x}\right)\left(Z_{r^{\prime}, s}^{n}-Z_{r, s}^{n}\right) d s \\
& +\int_{r}^{t} \nabla^{2} U_{n}\left(s, \gamma_{s, n}^{-1}\left(\tilde{X}_{s}^{n, x}\right)\right) \nabla \gamma_{s, n}^{-1}\left(\tilde{X}_{s}^{n, x}\right)\left(Z_{r^{\prime}, s}^{n}-Z_{r, s}^{n}\right) d B_{s} .
\end{aligned}
$$

By Lemma B.3 we know that $\nabla U_{n}$ is bounded uniformly in $n$ and Lemma B.2 shows that $\nabla^{2} U_{n}$ belongs, at least, to $L_{p}^{q}$ uniformly in $n$. This implies that the stochastic integral in the expression for $\left\|Z_{r^{\prime}, t}^{n}-Z_{r, t}^{n}\right\|^{\alpha}$ is a true martingale. As a result, since the initial condition $Z_{r^{\prime}, r}^{n}-Z_{r, r}^{n}$ is $\mathcal{F}_{r}$-measurable for each $n \geq 0$, for a given $\alpha \geq 2$, by Itô's formula we have

$$
\begin{aligned}
\left\|Z_{r^{\prime}, t}^{n}-Z_{r, t}^{n}\right\|^{\alpha} \lesssim & \left\|Z_{r^{\prime}, r}^{n}-Z_{r, r}^{n}\right\|^{\alpha}+\int_{r}^{t}\left\|Z_{r^{\prime}, s}^{n}-Z_{r, s}^{n}\right\|^{\alpha} d s+M_{t}^{n} \\
& +\int_{r}^{t}\left\|Z_{r^{\prime}, s}^{n}-Z_{r, s}^{n}\right\|^{\alpha-2} \operatorname{Tr}\left[\left(\nabla^{2} U_{n}\left(s, \gamma_{s, n}^{-1}\left(\tilde{X}_{s}^{n, x}\right)\right) \nabla \gamma_{s, n}^{-1}\left(\tilde{X}_{s}^{n, x}\right)\left(Z_{r^{\prime}, s}^{n}-Z_{r, s}^{n}\right)\right)\right. \\
& \left.\times\left(\nabla^{2} U_{n}\left(s, \gamma_{s, n}^{-1}\left(\tilde{X}_{s}^{n, x}\right)\right) \nabla \gamma_{s, n}^{-1}\left(\tilde{X}_{s}^{n, x}\right)\left(Z_{r^{\prime}, s}^{n}-Z_{r, s}^{n}\right)\right)^{*}\right] d s,
\end{aligned}
$$

where $M_{t}$ here denotes the martingale part after applying Itô's formua, $\operatorname{Tr}$ stands for the trace and $*$ for the transposition of matrices.

We proceed then using the fact that the trace of the matrix appearing in (20) can be bounded by a constant $C_{p, d}$ independent of $n$, times $\left\|Z_{r^{\prime}, s}^{n}-Z_{r, s}^{n}\right\|^{2}\left\|\nabla^{2} U_{n}\left(s, \gamma_{s, n}^{-1}\left(\tilde{X}_{s}^{n, x}\right)\right) \nabla \gamma_{s, n}^{-1}\left(\tilde{X}_{s}^{n, x}\right)\right\|^{2}$.

Altogether,

$$
\begin{aligned}
\left\|Z_{r^{\prime}, t}^{n}-Z_{r, t}^{n}\right\|^{\alpha} \lesssim & \left\|Z_{r^{\prime}, r}^{n}-Z_{r, r}^{n}\right\|^{\alpha}+\int_{r}^{t}\left\|Z_{r^{\prime}, s}^{n}-Z_{r, s}^{n}\right\|^{\alpha} d s+M_{t}^{n} \\
& +\int_{r}^{t}\left\|Z_{r^{\prime}, s}^{n}-Z_{r, s}^{n}\right\|^{\alpha}\left\|\nabla^{2} U_{n}\left(s, \gamma_{s, n}^{-1}\left(\tilde{X}_{s}^{n, x}\right)\right) \nabla \gamma_{s, n}^{-1}\left(\tilde{X}_{s}^{n, x}\right)\right\|^{2} d s .
\end{aligned}
$$

Consider thus the process

$$
V_{t}^{n}:=\int_{r}^{t}\left\|\nabla^{2} U_{n}\left(s, \gamma_{s, n}^{-1}\left(\tilde{X}_{s}^{n, x}\right)\right) \nabla \gamma_{s, n}^{-1}\left(\tilde{X}_{s}^{n, x}\right)\right\|^{2} d s .
$$

The process $V_{t}^{n}$ is a continuous non-decreasing and $\left\{\mathcal{F}_{t}\right\}_{t \in[0, T]}$-adapted process such that $V_{r}^{n}=0$. Then Lemma B.2 in connection with Theorem 2.2 we have that $\sup _{n \geq 0} E\left[V_{t}^{n}\right]<\infty$.

Then Itô's formula yields

$$
e^{-V_{t}^{n}}\left\|Z_{r^{\prime}, t}^{n}-Z_{r, t}^{n}\right\|^{\alpha} \lesssim\left\|Z_{r^{\prime}, r}^{n}-Z_{r, r}^{n}\right\|^{\alpha}+\int_{r}^{t} e^{-V_{s}^{n}}\left\|Z_{r^{\prime}, s}^{n}-Z_{r, s}^{n}\right\|^{\alpha} d s+\int_{r}^{t} e^{-V_{s}^{n}} d M_{s} .
$$

Then taking expectation

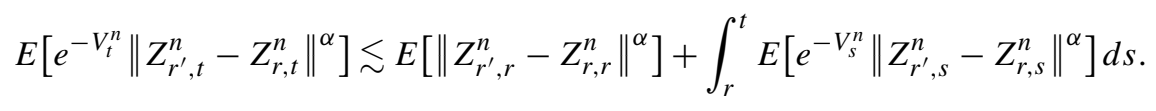

Then Gronwall's inequality gives

$$
E\left[e^{-V_{t}^{n}}\left\|Z_{r^{\prime}, t}^{n}-Z_{r, t}^{n}\right\|^{\alpha}\right] \lesssim E\left[\left\|Z_{r^{\prime}, r}^{n}-Z_{r, r}^{n}\right\|^{\alpha}\right] .
$$


At this point, it is easy to see, following similar steps, that for the process $Z_{r, t}^{n}$ one has

$$
E\left[e^{-V_{t}^{n}}\left\|Z_{r, t}^{n}\right\|^{\alpha}\right] \lesssim E\left[\left\|Z_{r, r}^{n}\right\|^{\alpha}\right]
$$

where $Z_{r, r}^{n}=\mathcal{I}_{d}+\nabla U_{n}\left(r, \gamma_{r, n}^{-1}\left(\tilde{X}_{r}^{n, x}\right)\right)$. So

$$
\sup _{n \geq 0} \sup _{r \in[0, T]} E\left[e^{-V_{t}^{n}}\left\|Z_{r, t}^{n}\right\|^{\alpha}\right] \lesssim 1+\sup _{n \geq 0} \sup _{r \in[0, T]} E\left[\left\|\nabla U_{n}\left(r, \gamma_{r, n}^{-1}\left(\tilde{X}_{r}^{n, x}\right)\right)\right\|^{\alpha}\right]<\infty
$$

because of Lemma B.3(ii) for a sufficiently large $\lambda \in \mathbb{R}$.

Then, the Cauchy-Schwarz inequality and Lemma B.5 give

$$
\sup _{n \geq 0} \sup _{r \in[0, T]} E\left[\left\|Z_{r, t}^{n}\right\|^{\alpha}\right] \leq \sup _{n \geq 0} \sup _{r \in[0, T]} E\left[e^{-2 V_{t}^{n}}\left\|Z_{r, t}^{n}\right\|^{2 \alpha}\right]^{1 / 2} \sup _{n \geq 0} E\left[e^{2 V_{T}^{n}}\right]^{1 / 2}<\infty .
$$

We continue to prove the estimate (17). Recall that

$$
\begin{aligned}
Z_{r^{\prime}, r}^{n}-Z_{r, r}^{n}= & \nabla U_{n}\left(r^{\prime}, \gamma_{r^{\prime}, n}^{-1}\left(\tilde{X}_{r^{\prime}}^{n, x}\right)\right)-\nabla U_{n}\left(r, \gamma_{r, n}^{-1}\left(\tilde{X}_{r}^{n, x}\right)\right) \\
& +\lambda \int_{r^{\prime}}^{r} \nabla U_{n}\left(s, \gamma_{s, n}^{-1}\left(\tilde{X}_{s}^{n, x}\right)\right) \nabla \gamma_{s, n}^{-1}\left(\tilde{X}_{s}^{n, x}\right) Z_{r^{\prime}, s} d s \\
& +\int_{r^{\prime}}^{r} \nabla^{2} U_{n}\left(s, \gamma_{s, n}^{-1}\left(\tilde{X}_{s}^{n, x}\right)\right) \nabla \gamma_{s, n}^{-1}\left(\tilde{X}_{s}^{n, x}\right) Z_{r^{\prime}, s} d B_{s} .
\end{aligned}
$$

Then taking norm and using Burkholder-Davis-Gundy inequality we get

$$
\begin{aligned}
E\left[\left\|Z_{r^{\prime}, r}^{n}-Z_{r, r}^{n}\right\|^{\alpha}\right] \lesssim & E\left[\left\|\nabla U_{n}\left(r^{\prime}, \gamma_{r^{\prime}, n}^{-1}\left(\tilde{X}_{r^{\prime}}^{n, x}\right)\right)-\nabla U_{n}\left(r, \gamma_{r, n}^{-1}\left(\tilde{X}_{r}^{n, x}\right)\right)\right\|^{\alpha}\right] \\
& +\lambda^{\alpha} E\left[\left(\int_{r^{\prime}}^{r}\left\|\nabla U_{n}\left(s, \gamma_{s, n}^{-1}\left(\tilde{X}_{s}^{n, x}\right)\right) \nabla \gamma_{s, n}^{-1}\left(\tilde{X}_{s}^{n, x}\right) Z_{r^{\prime}, s}\right\| d s\right)^{\alpha}\right] \\
& +E\left[\left(\int_{r^{\prime}}^{r}\left\|\nabla^{2} U_{n}\left(s, \gamma_{s, n}^{-1}\left(\tilde{X}_{s}^{n, x}\right)\right) \nabla \gamma_{s, n}^{-1}\left(\tilde{X}_{s}^{n, x}\right) Z_{r^{\prime}, s}\right\|^{2} d s\right)^{\alpha / 2}\right] . \\
= & :(i)_{n}+(i i)_{n}+(i i i)_{n} .
\end{aligned}
$$

The aim now is to find Hölder bounds in the sense of (17) for the expressions appearing in (28).

For $(i)_{n}$ we may write

$$
\begin{aligned}
(i)_{n}= & E\left[\left\|\nabla U_{n}\left(r^{\prime}, \gamma_{r^{\prime}, n}^{-1}\left(\tilde{X}_{r^{\prime}}^{n, x}\right)\right)-\nabla U_{n}\left(r, \gamma_{r, n}^{-1}\left(\tilde{X}_{r}^{n, x}\right)\right)\right\|^{\alpha}\right] \\
\lesssim & E\left[\left\|\nabla U_{n}\left(r^{\prime}, \gamma_{r^{\prime}, n}^{-1}\left(\tilde{X}_{r^{\prime}}^{n, x}\right)\right)-\nabla U_{n}\left(r, \gamma_{r^{\prime}, n}^{-1}\left(\tilde{X}_{r^{\prime}}^{n, x}\right)\right)\right\|^{\alpha}\right] \\
& +E\left[\left\|\nabla U_{n}\left(r, \gamma_{r^{\prime}, n}^{-1}\left(\tilde{X}_{r^{\prime}}^{n, x}\right)\right)-\nabla U_{n}\left(r, \gamma_{r, n}^{-1}\left(\tilde{X}_{r}^{n, x}\right)\right)\right\|^{\alpha}\right] .
\end{aligned}
$$

Then by Lemma 2.3 there exists an $\varepsilon \in(0,1 / \alpha)$, i.e. in fact we can choose $\varepsilon=(1-d / p-2 / q) / 3$, when $\alpha \in(2,3)$, and a constant $C_{p, q, d, \alpha}>0$ independent of $n \geq 0$ such that

$$
\begin{aligned}
& E\left[\left\|\nabla U_{n}\left(r^{\prime}, \gamma_{r^{\prime}, n}^{-1}\left(\tilde{X}_{r^{\prime}}^{n, x}\right)\right)-\nabla U_{n}\left(r, \gamma_{r^{\prime}, n}^{-1}\left(\tilde{X}_{r^{\prime}}^{n, x}\right)\right)\right\|^{\alpha}\right] \\
& \quad \leq C_{p, q, d, \alpha}\left(\left|r^{\prime}-r\right|^{\varepsilon / 2}\left\|\nabla U_{n}\right\|_{H_{2, p}^{q}}^{1-1 / q-\varepsilon / 2}\left\|\partial_{t} U_{n}\right\|_{L_{p}^{q}}^{1 / q+\varepsilon / 2}\right)^{\alpha}
\end{aligned}
$$

and

$$
\begin{aligned}
& E\left[\left\|\nabla U_{n}\left(r, \gamma_{r^{\prime}, n}^{-1}\left(\tilde{X}_{r^{\prime}}^{n, x}\right)\right)-\nabla U_{n}\left(r, \gamma_{r, n}^{-1}\left(\tilde{X}_{r}^{n, x}\right)\right)\right\|^{\alpha}\right] \\
& \quad \leq C_{p, q, d, \alpha} T^{-\alpha / q} E\left[\left|\gamma_{r^{\prime}, n}^{-1}\left(\tilde{X}_{r^{\prime}}^{n, x}\right)-\gamma_{r, n}^{-1}\left(\tilde{X}_{r}^{n, x}\right)\right|^{\alpha \varepsilon}\right]\left(\left\|U_{n}\right\|_{H_{2, p}^{q}}+T\left\|\partial_{t} U_{n}\right\|_{L_{p}^{q}}\right)^{\alpha} .
\end{aligned}
$$


The above bounds in connection with inequality (11) in Theorem 2.2 give

$$
(i)_{n} \leq C_{p, q, d, \alpha, T}\left(\left\|b_{n}\right\|_{L_{p}^{q}}\right)\left(\left|r^{\prime}-r\right|^{\alpha \varepsilon / 2}+E\left[\left|\gamma_{r^{\prime}, n}^{-1}\left(\tilde{X}_{r^{\prime}}^{n, x}\right)-\gamma_{r, n}^{-1}\left(\tilde{X}_{r}^{n, x}\right)\right|^{\alpha \varepsilon}\right]\right)
$$

for some continuous function $C_{p, q, d, \alpha, T}(\cdot)$ and hence

$$
\sup _{n \geq 0} C_{p, q, d, \alpha, T}\left(\left\|b_{n}\right\|_{L_{p}^{q}}\right)<\infty .
$$

Moreover, using Girsanov's theorem, we obtain that

$$
\begin{aligned}
E\left[\left|\gamma_{r^{\prime}, n}^{-1}\left(\tilde{X}_{r^{\prime}}^{n, x}\right)-\gamma_{r, n}^{-1}\left(\tilde{X}_{r}^{n, x}\right)\right|\right] & =E\left[\left|X_{r^{\prime}}^{n, x}-X_{r}^{n, x}\right|\right] \\
& \lesssim E\left[\left|\int_{r^{\prime}}^{r} b_{n}\left(s, x+B_{s}\right) d s\right| \mathcal{E}\left(\int_{0}^{T} b_{n}\left(u, x+B_{u}\right) d B_{u}\right)\right]+E\left[\left|B_{r^{\prime}}-B_{r}\right|\right] \\
& \lesssim\left|r^{\prime}-r\right|^{1 / 2} E\left[\int_{r^{\prime}}^{r}\left|b_{n}\left(s, x+B_{s}\right)\right|^{2} d s\right]^{1 / 2}+\left|r^{\prime}-r\right|^{1 / 2} \\
& \lesssim\left|r^{\prime}-r\right|^{1 / 2},
\end{aligned}
$$

where $\mathcal{E}\left(M_{t}\right)$ denotes the Doléans-Dade exponential of a martingale $M$ and we used, Cauchy-Schwarz inequality and both that

$$
\sup _{n \geq 0} E\left[\mathcal{E}\left(\int_{0}^{T} b_{n}\left(u, x+B_{u}\right) d B_{u}\right)^{2}\right]<\infty
$$

and

$$
\sup _{n \geq 0} E\left[\int_{r^{\prime}}^{r}\left|b_{n}\left(s, x+B_{s}\right)\right|^{2} d s\right]^{1 / 2}<\infty,
$$

see [20, Lemma 3.2] or Lemma B.1.

By Jensen's inequality for concave functions and the previous estimate we have

$$
E\left[\left|\gamma_{r^{\prime}, n}^{-1}\left(\tilde{X}_{r^{\prime}}^{n, x}\right)-\gamma_{r, n}^{-1}\left(\tilde{X}_{r}^{n, x}\right)\right|^{\alpha \varepsilon}\right] \leq E\left[\left|\gamma_{r^{\prime}, n}^{-1}\left(\tilde{X}_{r^{\prime}}^{n, x}\right)-\gamma_{r, n}^{-1}\left(\tilde{X}_{r}^{n, x}\right)\right|\right]^{\alpha \varepsilon} \lesssim\left|r^{\prime}-r\right|^{\alpha \varepsilon / 2} .
$$

Altogether,

$$
(i)_{n} \leq C_{p, q, d, \alpha, T}\left(\left\|b_{n}\right\|_{L_{p}^{q}}\right)\left|r^{\prime}-r\right|^{\delta}
$$

for a $\delta \in(0,1)$.

For the second term, (ii) $)_{n}$, we use Hölder's inequality, Lemma B.3(ii) for a sufficiently large $\lambda \in \mathbb{R}$, Lemma B.4 and the estimate (18) to obtain

$$
\begin{aligned}
(i i)_{n} & \lesssim \lambda^{\alpha}\left|r^{\prime}-r\right|^{\alpha-1}\left(\sup _{s \in[0, t]} E\left[\left\|\nabla U_{n}\left(s, \gamma_{s, n}^{-1}\left(\tilde{X}_{s}^{n, x}\right)\right) \nabla \gamma_{s, n}^{-1}\left(\tilde{X}_{s}^{n, x}\right)\right\|^{2 \alpha}\right] d s\right)^{1 / 2}\left(\int_{r^{\prime}}^{r} E\left[\left\|Z_{r^{\prime}, s}^{n}\right\|^{2 \alpha}\right] d s\right)^{1 / 2} \\
& \leq C_{p, q, d, \alpha, T}\left|r^{\prime}-r\right|^{\delta}
\end{aligned}
$$

for a $\delta \in(0,1)$.

Finally, for the third term, for $\alpha \geq 2$, we use Hölder's inequality to obtain

$$
(\text { iii })_{n} \lesssim\left|r^{\prime}-r\right|^{\frac{\alpha-2}{2}} E\left[\int_{r^{\prime}}^{r}\left\|\nabla^{2} U_{n}\left(s, \gamma_{s, n}^{-1}\left(\tilde{X}_{s}^{n}\right)\right)\right\|^{\alpha}\left\|\nabla \gamma_{s, n}^{-1}\left(\tilde{X}_{s}^{n}\right)\right\|^{\alpha}\left\|Z_{r^{\prime}, s}^{n}\right\|^{\alpha} d s\right] .
$$


Then choose $\alpha=2(1+\delta)$ with $\delta \in(0,1 / 4)$ and use Lemma B.4 to get

$$
(\text { iii })_{n} \lesssim\left|r^{\prime}-r\right|^{\delta} E\left[\int_{r^{\prime}}^{r}\left\|\nabla^{2} U_{n}\left(s, \gamma_{s, n}^{-1}\left(\tilde{X}_{s}^{n}\right)\right)\right\|^{2(1+\delta)}\left\|Z_{r^{\prime}, s}^{n}\right\|^{2(1+\delta)} d s\right] .
$$

Then Fubini's theorem, Hölder's inequality once more with respect to $\mu(d \omega)$, with exponent $1+\delta^{\prime}, \delta^{\prime} \in(0,1 / 4)$ and Cauchy-Schwarz' inequality yield

$$
\begin{aligned}
& E\left[\int_{r^{\prime}}^{r}\left\|\nabla^{2} U_{n}\left(s, \gamma_{s, n}^{-1}\left(\tilde{X}_{s}^{n}\right)\right)\right\|^{2(1+\delta)}\left\|Z_{r^{\prime}, s}^{n}\right\|^{2(1+\delta)} d s\right] \\
& \quad=\int_{r^{\prime}}^{r} E\left[\left\|\nabla^{2} U_{n}\left(s, \gamma_{s, n}^{-1}\left(\tilde{X}_{s}^{n}\right)\right)\right\|^{2(1+\delta)}\left\|Z_{r^{\prime}, s}^{n}\right\|^{2(1+\delta)}\right] d s \\
& \quad \lesssim \int_{r^{\prime}}^{r} E\left[\left\|\nabla^{2} U_{n}\left(s, \gamma_{s, n}^{-1}\left(\tilde{X}_{s}^{n}\right)\right)\right\|^{2(1+\delta)\left(1+\delta^{\prime}\right)}\right]^{1 /\left(1+\delta^{\prime}\right)} E\left[\left\|Z_{r^{\prime}, s}^{n}\right\|^{2(1+\delta) \frac{1+\delta^{\prime}}{\delta^{\prime}}}\right]^{\frac{\delta^{\prime}}{1+\delta^{\prime}}} d s \\
& \quad \lesssim \sup _{n \geq 0} \sup _{s \in\left[r^{\prime}, r\right]} E\left[\left\|Z_{r^{\prime}, s}^{n}\right\|^{2(1+\delta) \frac{1+\delta^{\prime}}{\delta^{\prime}}}\right]^{\frac{\delta^{\prime}}{1+\delta^{\prime}}} \int_{r^{\prime}}^{r} E\left[\left\|\nabla^{2} U_{n}\left(s, \gamma_{s, n}^{-1}\left(\tilde{X}_{s}^{n}\right)\right)\right\|^{2(1+\delta)\left(1+\delta^{\prime}\right)}\right]^{1 /\left(1+\delta^{\prime}\right)} d s \\
& \quad \lesssim \int_{0}^{T} E\left[\left\|\nabla^{2} U_{n}\left(s, \gamma_{s, n}^{-1}\left(\tilde{X}_{s}^{n}\right)\right)\right\|^{2(1+\delta)\left(1+\delta^{\prime}\right)}\right]^{1 /\left(1+\delta^{\prime}\right)} d s,
\end{aligned}
$$

where the last step follows from (18). For the last factor, since $0<1 /\left(1+\delta^{\prime}\right)<1$, using the inverse Jensen's inequality and the fact that $1<(1+\delta)\left(1+\delta^{\prime}\right)<2$ for suitable $\delta, \delta^{\prime} \in(0,1 / 4)$ in connection with Lemma B.2 we have

$$
\begin{aligned}
& \int_{0}^{T} E\left[\left\|\nabla^{2} U_{n}\left(s, \gamma_{s, n}^{-1}\left(\tilde{X}_{s}^{n}\right)\right)\right\|^{2(1+\delta)\left(1+\delta^{\prime}\right)}\right]^{1 /\left(1+\delta^{\prime}\right)} d s \\
& \quad \leq T^{1-1 /\left(1+\delta^{\prime}\right)}\left(E\left[\int_{0}^{T}\left\|\nabla^{2} U_{n}\left(s, \gamma_{s, n}^{-1}\left(\tilde{X}_{s}^{n}\right)\right)\right\|^{2(1+\delta)\left(1+\delta^{\prime}\right)} d s\right]\right)^{1 /\left(1+\delta^{\prime}\right)} \leq M<\infty
\end{aligned}
$$

for every $n \geq 0$, w.r.t. a constant $M$.

As a summary, it follows from (25) that

$$
E\left[e^{-V_{t}^{n}}\left\|Z_{r^{\prime}, t}^{n}-Z_{r, t}^{n}\right\|^{2(1+\delta)}\right] \leq C_{p, q, d, \alpha, T}\left(\left\|b_{n}\right\|_{L_{p}^{q}}\right)\left|r^{\prime}-r\right|^{\delta} .
$$

Then by Hölder's inequality with exponent $1+\delta, \delta \in(0,1)$ together with Lemma B.5 we obtain

$$
\begin{aligned}
E\left[\left\|Z_{r^{\prime}, t}^{n}-Z_{r, t}^{n}\right\|^{2}\right] & =E\left[e^{\frac{1}{1+\delta} V_{t}^{n}} e^{-\frac{1}{1+\delta} V_{t}^{n}}\left\|Z_{r^{\prime}, t}^{n}-Z_{r, t}^{n}\right\|^{2}\right] \\
& \leq E\left[e^{\frac{1}{\delta} V_{t}^{n}}\right]^{\frac{\delta}{1+\delta}} E\left[e^{-V_{t}^{n}}\left\|Z_{r^{\prime}, t}^{n}-Z_{r, t}^{n}\right\|^{2(1+\delta)}\right]^{\frac{1}{1+\delta}} \\
& \leq C_{p, q, d, \alpha, T}\left(\left\|b_{n}\right\|_{L_{p}^{q}}\right)\left|r^{\prime}-r\right|^{\delta /(1+\delta)}
\end{aligned}
$$

with

$$
\sup _{n \geq 0} C_{p, q, d, \alpha, T}\left(\left\|b_{n}\right\|_{L_{p}^{q}}\right)<\infty .
$$

Remark 2.7. The bound given in (18) is in fact uniform in $x \in \mathbb{R}^{d}$. Indeed, by Lemma B.3 item (ii) we have that the bound given in (26) is also uniform in $x \in \mathbb{R}^{d}$. Moreover, since $\Delta U_{n} \in L_{p}^{q}$ for all $n \geq 0$, then by Lemma B.3 item (iii) in connection with Lemma B. 1 we have that for any $k \in \mathbb{R}$

$$
\sup _{x \in \mathbb{R}^{d}} \sup _{n \geq 0} E\left[e^{k V_{T}^{n}}\right]<\infty .
$$


Hence, for any $\alpha \geq 1$

$$
\sup _{x \in \mathbb{R}^{d}} \sup _{r \in[0, T]} \sup _{n \geq 0} E\left[\left\|D_{r} X_{t}^{n, x}\right\|^{\alpha}\right]<\infty .
$$

Remark 2.8. One also directly checks that the same holds for the spatial derivatives, that is for any $\alpha \geq 1$

$$
\sup _{x \in \mathbb{R}^{d}} \sup _{n \geq 0} E\left[\left\|\frac{\partial}{\partial x} X_{t}^{n, x}\right\|^{\alpha}\right]<\infty
$$

by using the fact that $\frac{\partial}{\partial x} X_{t}^{n, x}$ solves the same SDE as $D_{r} X_{t}^{n, x}$, starting at $r=0$.

As a repercussion of Theorem 2.6 we have the following result which is central in the proof of the existence of strong solutions of (5).

Corollary 2.9. Let $\left\{b_{n}\right\}_{n \geq 0}$ be a sequence of compactly supported smooth functions approximating $b$ in $L_{p}^{q}$. Denote, as before, $X_{t}^{x, n}$ the solution to equation (5) with drift coefficient $b_{n}$. Then for each $t \in[0, T]$ the sequence of random variables $X_{t}^{n, x}, n \geq 0$ is relatively compact in $L^{2}(\Omega)$.

Proof. This is a direct consequence of the compactness criterion that can be found in Appendix C, Lemma C.1 and C.2, which is due to [4], together with Theorem 2.6. One can check that the double integral in Lemma C.2 is finite. Namely

$$
\int_{0}^{T} \int_{0}^{T} \frac{E\left[\left\|Z_{r^{\prime}, t}^{n}-Z_{r, t}^{n}\right\|^{2}\right]}{\left|r^{\prime}-r\right|^{1+2 \beta}} d r^{\prime} d r \leq \int_{0}^{T} \int_{0}^{T} \frac{1}{\left|r^{\prime}-r\right|^{2 \beta+1-\delta}} d r^{\prime} d r<\infty
$$

for any $0<\delta<1$ and $2 \beta+1-\delta<1$.

Next, we turn to the second step of our scheme. The following lemma gives a criterion under which the process $Y_{t}^{b}$ belongs to the Hida distribution space.

Lemma 2.10. Suppose that

$$
E_{\mu}\left[\exp \left(36 \int_{0}^{T}\left|b\left(s, B_{s}\right)\right|^{2} d s\right)\right]<\infty
$$

where the drift $b:[0,1] \times \mathbb{R}^{d} \longrightarrow \mathbb{R}^{d}$ is measurable (in particular, (29) is valid for $b \in L_{p}^{q}$ because of Lemma (B.1)). Then the coordinates of the process $Y_{t}^{b}$, defined in (16), that is

$$
Y_{t}^{i, b}=E_{\widetilde{\mu}}\left[\widetilde{B}_{t}^{(i)} \mathcal{E}_{T}^{\diamond}(b)\right],
$$

are elements of the Hida distribution space.

Proof. See [27] for a similar proof.

The following lemma is a generalized version of Lemma 12 in [27] in the case of bounded coefficients, which now is adapted to the case of vector fields in $L_{p}^{q}$.

Lemma 2.11. Let $\varepsilon \in(0,1)$ and define $p_{\varepsilon}:=1+\varepsilon$ and $q_{\varepsilon}:=\frac{1+\varepsilon}{\varepsilon}$. Let $b_{n}:[0, T] \times \mathbb{R}^{d} \longrightarrow \mathbb{R}^{d}$ be a sequence of Borel measurable functions such that

$$
\sup _{n \geq 0} E\left[\exp \left(16 q_{\varepsilon}\left(8 q_{\varepsilon}-1\right) \int_{0}^{T}\left|b_{n}\left(s, B_{s}\right)\right|^{2} d s\right)\right]<\infty
$$


holds. Then

$$
\left|S\left(Y_{t}^{i, b_{n}}-Y_{t}^{i, b}\right)(\phi)\right| \leq \mathrm{const} \cdot E\left[J_{n}\right]^{\frac{1}{p_{\varepsilon}}} \cdot \exp \left(2\left(8 q_{\varepsilon}-1\right) \int_{0}^{T}|\phi(s)|^{2} d s\right)
$$

for all $\phi \in\left(\mathcal{S}_{\mathbb{C}}([0,1])\right)^{d}, i=1, \ldots, d$, where $S$ denotes the $S$-transform (see Section A.1 in Appendix A) and where the factor $J_{n}$ is defined by

$$
J_{n}=\sum_{j=1}^{d} 2\left|\int_{0}^{T}\left(b_{n}^{(j)}\left(s, \tilde{B}_{s}^{(j)}\right)-b^{(j)}\left(s, \tilde{B}_{s}^{(j)}\right)\right)^{2} d s\right|^{\frac{p_{\varepsilon}}{2}}+\left|\int_{0}^{T}\left(b^{(j)}\left(s, \tilde{B}_{s}^{(j)}\right)^{2}-b_{n}^{(j)}\left(s, \tilde{B}_{s}^{(j)}\right)^{2}\right) d s\right|^{p_{\varepsilon}} .
$$

Here $\mathcal{S}_{\mathbb{C}}([0,1])$ is the complexification of the Schwarz space $\mathcal{S}([0,1])$ on $[0,1]$, see Section A.1 in Appendix A.

In particular, if $b_{n}$ approximates $b$ in the following sense

$$
E\left[J_{n}\right] \rightarrow 0
$$

as $n \rightarrow \infty$, it follows that

$$
Y_{t}^{b_{n}} \rightarrow Y_{t}^{b} \quad \text { in }(\mathcal{S})^{*}
$$

as $n \rightarrow \infty$ for all $0 \leq t \leq T, i=1, \ldots, d$.

Proof. For $i=1, \ldots, d$ we obtain by Proposition 2.4 and (59) that

$$
\begin{aligned}
\left|S\left(Y_{t}^{i, b_{n}}-Y_{t}^{i, b}\right)(\phi)\right| \leq & E_{\tilde{\mu}}\left[| \tilde { B } _ { t } ^ { ( i ) } | \operatorname { e x p } \left\{\sum _ { j = 1 } ^ { d } \operatorname { R e } \left[\int_{0}^{T}\left(b^{(j)}\left(s, \tilde{B}_{s}^{(j)}\right)+\phi^{(j)}(s)\right) d \tilde{B}_{s}^{(j)}\right.\right.\right. \\
& \left.\left.-\frac{1}{2} \int_{0}^{T}\left(b^{(j)}\left(s, \tilde{B}_{s}^{(j)}\right)+\phi^{(j)}(s)\right)^{2} d s\right]\right\} \\
& \times \mid \exp \left\{\sum_{j=1}^{d} \int_{0}^{T}\left(b_{n}^{(j)}\left(s, \tilde{B}_{s}^{(j)}\right)-b^{(j)}\left(s, \tilde{B}_{s}^{(j)}\right)\right) d \tilde{B}_{s}^{(j)}\right. \\
& +\frac{1}{2} \int_{0}^{T}\left(b^{(j)}\left(s, \tilde{B}_{s}^{(j)}\right)^{2}-b_{n}^{(j)}\left(s, \tilde{B}_{s}^{(j)}\right)^{2}\right) d s \\
& \left.\left.+\int_{0}^{T} \phi^{(j)}(s)\left(b^{(j)}\left(s, \tilde{B}_{s}^{(j)}\right)-b_{n}^{(j)}\left(s, \tilde{B}_{s}^{(j)}\right)\right) d s\right\}-1 \mid\right] .
\end{aligned}
$$

Since $|\exp \{z\}-1| \leq|z| \exp \{|z|\}$ it follows from Hölder's inequality with exponents $p_{\varepsilon}=1+\varepsilon$ and $q_{\varepsilon}=\frac{1+\varepsilon}{\varepsilon}$, for an appropriate $\varepsilon>0$, that

$$
\begin{aligned}
\left|S\left(Y_{t}^{i, b_{n}}-Y_{t}^{i, b}\right)(\phi)\right| \leq & E_{\tilde{\mu}}\left[\left|Q_{n}\right|^{p_{\varepsilon}}\right]^{\frac{1}{p_{\varepsilon}}} E_{\tilde{\mu}}\left[\left(| \tilde { B } _ { t } ^ { ( i ) } | \operatorname { e x p } \left\{\sum _ { j = 1 } ^ { d } \operatorname { R e } \left[\int_{0}^{T}\left(b^{(j)}\left(s, \tilde{B}_{s}^{(j)}\right)+\phi^{(j)}(s)\right) d \tilde{B}_{s}^{(j)}\right.\right.\right.\right. \\
& \left.\left.\left.\left.-\frac{1}{2} \int_{0}^{T}\left(b^{(j)}\left(s, \tilde{B}_{s}^{(j)}\right)+\phi^{(j)}(s)\right)^{2} d s\right]\right\}\right)^{q_{\varepsilon}} \exp \left\{q_{\varepsilon}\left|Q_{n}\right|\right\}\right]^{\frac{1}{q_{\varepsilon}}}
\end{aligned}
$$

where

$$
\begin{aligned}
Q_{n}= & \sum_{j=1}^{d} \int_{0}^{T}\left(b_{n}^{(j)}\left(s, \tilde{B}_{s}^{(j)}\right)-b^{(j)}\left(s, \tilde{B}_{s}^{(j)}\right)\right) d \tilde{B}_{s}^{(j)}+\frac{1}{2} \int_{0}^{T}\left(b^{(j)}\left(s, \tilde{B}_{s}^{(j)}\right)^{2}-b_{n}^{(j)}\left(s, \tilde{B}_{s}^{(j)}\right)^{2}\right) d s \\
& +\int_{0}^{T} \phi^{(j)}(s)\left(b^{(j)}\left(s, \tilde{B}_{s}^{(j)}\right)-b_{n}^{(j)}\left(s, \tilde{B}_{s}^{(j)}\right)\right) d s .
\end{aligned}
$$


Then using the Cauchy-Schwarz inequality on the last integral and the fact that $|x| \leq e^{x}$ and $1 \leq e^{x}$ for $x \geq 0$ we may write

$$
\begin{aligned}
E_{\tilde{\mu}}\left[\left|Q_{n}\right|^{p_{\varepsilon}}\right] \leq & C \exp \left\{\left(\int_{0}^{T}|\phi(s)|^{2} d s\right)^{p_{\varepsilon} / 2}\right\} E_{\tilde{\mu}}\left[\sum_{j=1}^{d}\left|\int_{0}^{T}\left(b_{n}^{(j)}\left(s, \tilde{B}_{s}^{(j)}\right)-b^{(j)}\left(s, \tilde{B}_{s}^{(j)}\right)\right) d \tilde{B}_{s}^{(j)}\right|^{p_{\varepsilon}}\right. \\
& +\left|\frac{1}{2} \int_{0}^{T}\left(b^{(j)}\left(s, \tilde{B}_{s}^{(j)}\right)^{2}-b_{n}^{(j)}\left(s, \tilde{B}_{s}^{(j)}\right)^{2}\right) d s\right| \\
& \left.+\left|\int_{0}^{T}\left(b^{(j)}\left(s, \tilde{B}_{s}^{(j)}\right)-b_{n}^{(j)}\left(s, \tilde{B}_{s}^{(j)}\right)\right)^{2} d s\right|^{\frac{p_{\varepsilon}}{2}}\right] \\
= & C \exp \left\{\left(\int_{0}^{T}|\phi(s)|^{2} d s\right)^{p_{\varepsilon} / 2}\right\} E_{\tilde{\mu}}\left[\sum_{j=1}^{d} 2\left|\int_{0}^{T}\left(b_{n}^{(j)}\left(s, \tilde{B}_{s}^{(j)}\right)-b^{(j)}\left(s, \tilde{B}_{s}^{(j)}\right)\right)^{2} d s\right|^{\frac{p_{\varepsilon}}{2}}\right. \\
& +\left|\int_{0}^{T}\left(b^{(j)}\left(s, \tilde{B}_{s}^{(j)}\right)^{2}-b_{n}^{(j)}\left(s, \tilde{B}_{s}^{(j)}\right)^{2}\right) d s\right|
\end{aligned}
$$

where in the last inequality we used the Burkholder-Davis-Gundy inequality for the stochastic integral. Then

$$
E_{\tilde{\mu}}\left[\left|Q_{n}\right|^{p_{\varepsilon}}\right]^{\frac{1}{p_{\varepsilon}}} \leq C \exp \left\{\frac{1}{p_{\varepsilon}}\left(\int_{0}^{T}|\phi(s)|^{2} d s\right)^{p_{\varepsilon} / 2}\right\} E_{\tilde{\mu}}\left[J_{n}\right]^{\frac{1}{p_{\varepsilon}}}
$$

where

$$
J_{n}=\sum_{j=1}^{d} 2\left|\int_{0}^{T}\left(b_{n}^{(j)}\left(s, \tilde{B}_{s}^{(j)}\right)-b^{(j)}\left(s, \tilde{B}_{s}^{(j)}\right)\right)^{2} d s\right|^{\frac{p_{\varepsilon}}{2}}+\left|\int_{0}^{T}\left(b^{(j)}\left(s, \tilde{B}_{s}^{(j)}\right)^{2}-b_{n}^{(j)}\left(s, \tilde{B}_{s}^{(j)}\right)^{2}\right) d s\right|^{p_{\varepsilon}} .
$$

Further we get that

$$
\begin{aligned}
E_{\tilde{\mu}}\left[\left(| \tilde { B } _ { t } ^ { ( i ) } | \operatorname { e x p } \left\{\sum _ { j = 1 } ^ { d } \operatorname { R e } \left[\int_{0}^{T}\left(b^{(j)}\left(s, \tilde{B}_{s}^{(j)}\right)+\phi^{(j)}(s)\right) d \tilde{B}_{s}^{(j)}\right.\right.\right.\right. \\
\left.\left.\left.\left.\quad-\frac{1}{2} \int_{0}^{T}\left(b^{(j)}\left(s, \tilde{B}_{s}^{(j)}\right)+\phi^{(j)}(s)\right)^{2} d s\right]\right\}\right)^{q_{\varepsilon}} \exp \left\{q_{\varepsilon}\left|Q_{n}\right|\right\}\right]^{\frac{1}{q_{\varepsilon}}} \\
\leq E_{\tilde{\mu}}\left[\left(| \tilde { B } _ { t } ^ { ( i ) } | \operatorname { e x p } \left\{\sum _ { j = 1 } ^ { d } \operatorname { R e } \left[\int_{0}^{T}\left(b^{(j)}\left(s, \tilde{B}_{s}^{(j)}\right)+\phi^{(j)}(s)\right) d \tilde{B}_{s}^{(j)}\right.\right.\right.\right. \\
\left.\left.\left.\left.\quad-\frac{1}{2} \int_{0}^{T}\left(b^{(j)}\left(s, \tilde{B}_{s}^{(j)}\right)+\phi^{(j)}(s)\right)^{2} d s\right]\right\}\right)^{2 q_{\varepsilon}}\right]^{\frac{1}{2 q_{\varepsilon}}} E_{\tilde{\mu}}\left[\exp \left\{2 q_{\varepsilon}\left|Q_{n}\right|\right\}\right]^{\frac{1}{2 q_{\varepsilon}}} .
\end{aligned}
$$

Then for $z \in \mathbb{C}$ one has $\exp \{|z|\} \leq \frac{1}{2}(\exp \{2 \operatorname{Re} z\}+\exp \{-2 \operatorname{Re} z\}+\exp \{2 \operatorname{Im} z\}+\exp \{-2 \operatorname{Im} z\})$. Thus

$$
\begin{aligned}
E_{\tilde{\mu}}\left[\exp \left\{2 q_{\varepsilon}\left|Q_{n}\right|\right\}\right]^{\frac{1}{2 q_{\varepsilon}}} \leq & \frac{1}{2^{2 q_{\varepsilon}}}\left(E_{\tilde{\mu}}\left[\exp \left\{4 q_{\varepsilon} \operatorname{Re} Q_{n}\right\}\right]^{\frac{1}{2 q_{\varepsilon}}}+E_{\tilde{\mu}}\left[\exp \left\{-4 q_{\varepsilon} \operatorname{Re} Q_{n}\right\}\right]^{\frac{1}{2 q_{\varepsilon}}}\right. \\
& \left.+E_{\tilde{\mu}}\left[\exp \left\{4 q_{\varepsilon} \operatorname{Im} Q_{n}\right\}\right]^{\frac{1}{2 q_{\varepsilon}}}+E_{\tilde{\mu}}\left[\exp \left\{-4 q_{\varepsilon} \operatorname{Im} Q_{n}\right\}\right]^{\frac{1}{2 q_{\varepsilon}}}\right) .
\end{aligned}
$$


By the Cauchy-Schwarz inequality and the supermartingale property of Doléans-Dade exponentials we get

$$
\begin{aligned}
E_{\tilde{\mu}}\left[\exp \left\{4 q_{\varepsilon} \operatorname{Re} Q_{n}\right\}\right] \leq & E_{\tilde{\mu}}\left[\operatorname { e x p } \left\{\sum_{j=1}^{d} 32 q_{\varepsilon}^{2} \int_{0}^{T}\left(b_{n}^{(j)}\left(s, \tilde{B}_{s}^{(j)}\right)-b^{(j)}\left(s, \tilde{B}_{s}^{(j)}\right)\right)^{2} d s\right.\right. \\
& +4 q_{\varepsilon} \int_{0}^{T}\left(b^{(j)}\left(s, \tilde{B}_{s}^{(j)}\right)^{2}-b_{n}^{(j)}\left(s, \tilde{B}_{s}^{(j)}\right)^{2}\right) d s \\
& \left.\left.+8 q_{\varepsilon} \int_{0}^{T} \operatorname{Re} \phi^{(j)}(s)\left(b^{(j)}\left(s, \tilde{B}_{s}^{(j)}\right)-b_{n}^{(j)}\left(s, \tilde{B}_{s}^{(j)}\right)\right) d s\right\}\right]^{\frac{1}{2}} \\
\leq & L_{n} \exp \left\{2 q_{\varepsilon} \int_{0}^{T}|\phi(s)|^{2} d s\right\},
\end{aligned}
$$

where the last step follows from the fact that $\langle f, g\rangle \leq \frac{1}{2}\left(\|f\|^{2}+\|g\|^{2}\right), f, g \in L^{2}([0, T])$ and where

$$
\begin{aligned}
L_{n}= & E_{\tilde{\mu}}\left[\operatorname { e x p } \left\{\sum_{j=1}^{d} 4 q_{\varepsilon}\left(8 q_{\varepsilon}+1\right) \int_{0}^{T}\left(b_{n}^{(j)}\left(s, \tilde{B}_{s}^{(j)}\right)-b^{(j)}\left(s, \tilde{B}_{s}^{(j)}\right)\right)^{2} d s\right.\right. \\
& \left.\left.+4 q_{\varepsilon} \int_{0}^{T}\left(b^{(j)}\left(s, \tilde{B}_{s}^{(j)}\right)^{2}-b_{n}^{(j)}\left(s, \tilde{B}_{s}^{(j)}\right)^{2}\right) d s\right\}\right]^{\frac{1}{2}} .
\end{aligned}
$$

Similarly, one also obtains

$$
E_{\tilde{\mu}}\left[\exp \left\{-4 q_{\varepsilon} \operatorname{Re} Q_{n}\right\}\right] \leq L_{n} \exp \left\{2 q_{\varepsilon} \int_{0}^{T}|\phi(s)|^{2} d s\right\}
$$

In the same way, one also obtains the same bounds for $E_{\tilde{\mu}}\left[\exp \left\{4 q_{\varepsilon} \operatorname{Im} Q_{n}\right\}\right]$ and $E_{\tilde{\mu}}\left[\exp \left\{-4 q_{\varepsilon} \operatorname{Im} Q_{n}\right\}\right]$. Finally, for the remaining factor we see that

$$
\begin{aligned}
& E_{\tilde{\mu}}\left[\left(| \tilde { B } _ { t } ^ { ( i ) } | \operatorname { e x p } \left\{\sum _ { j = 1 } ^ { d } \operatorname { R e } \left[\int_{0}^{T}\left(b^{(j)}\left(s, \tilde{B}_{s}^{(j)}\right)+\phi^{(j)}(s)\right) d \tilde{B}_{s}^{(j)}\right.\right.\right.\right. \\
&\left.\left.\left.\left.-\frac{1}{2} \int_{0}^{T}\left(b^{(j)}\left(s, \tilde{B}_{s}^{(j)}\right)+\phi^{(j)}(s)\right)^{2} d s\right]\right\}\right)^{2 q_{\varepsilon}}\right]^{\frac{1}{2 q_{\varepsilon}}} \\
& \leq E_{\tilde{\mu}}\left[\left|\tilde{B}_{t}^{(i)}\right|^{4 q_{\varepsilon}}\right]^{\frac{1}{4 q_{\varepsilon}}} E_{\tilde{\mu}}\left[\operatorname { e x p } \left\{4 q _ { \varepsilon } \sum _ { j = 1 } ^ { d } \operatorname { R e } \left[\int_{0}^{T}\left(b^{(j)}\left(s, \tilde{B}_{s}^{(j)}\right)+\phi^{(j)}(s)\right) d \tilde{B}_{s}^{(j)}\right.\right.\right. \\
&\left.\left.\left.-\frac{1}{2} \int_{0}^{T}\left(b^{(j)}\left(s, \tilde{B}_{S}^{(j)}\right)+\phi^{(j)}(s)\right)^{2} d s\right]\right\}\right]^{\frac{1}{4 q_{\varepsilon}}} \\
& \leq E_{\tilde{\mu}}\left[\left|\tilde{B}_{t}^{(i)}\right|^{4 q_{\varepsilon}}\right]^{\frac{1}{4 q_{\varepsilon}}} E_{\tilde{\mu}}\left[\exp \left\{\sum_{j=1}^{d} 4 q_{\varepsilon}\left(8 q_{\varepsilon}-1\right) \int_{0}^{T} \operatorname{Re}\left(b^{(j)}\left(s, \tilde{B}_{s}^{(j)}\right)+\phi^{(j)}(s)\right)^{2} d s\right\}\right]^{\frac{1}{4 q_{\varepsilon}}} .
\end{aligned}
$$


Now, since $\operatorname{Re}\left(z^{2}\right) \leq(\operatorname{Re} z)^{2}, z \in \mathbb{C}$ we have that $\operatorname{Re}(b+\phi)^{2} \leq(b+\operatorname{Re} \phi)^{2}$ then using Minkowski's inequality, i.e. $\|f+g\|_{p}^{p} \leq 2^{p-1}\left(\|f\|_{p}^{p}+\|g\|_{p}^{p}\right)$ for any $p \geq 1$ and Cauchy-Schwarz inequality w.r.t. $\tilde{\mu}$ one finally obtains

$$
\begin{aligned}
& E_{\tilde{\mu}}\left[\left(| \tilde { B } _ { t } ^ { ( i ) } | \operatorname { e x p } \left\{\sum _ { j = 1 } ^ { d } \operatorname { R e } \left[\int_{0}^{T}\left(b^{(j)}\left(s, \tilde{B}_{s}^{(j)}\right)+\phi^{(j)}(s)\right) d \tilde{B}_{s}^{(j)}\right.\right.\right.\right. \\
&\left.\left.\left.\left.-\frac{1}{2} \int_{0}^{T}\left(b^{(j)}\left(s, \tilde{B}_{s}^{(j)}\right)+\phi^{(j)}(s)\right)^{2} d s\right]\right\}\right)^{2 q_{\varepsilon}}\right]^{\frac{1}{2 q_{\varepsilon}}} \\
& \leq C E_{\tilde{\mu}}\left[\exp \left\{16 q_{\varepsilon}\left(8 q_{\varepsilon}-1\right) \int_{0}^{T}\left|b\left(s, \tilde{B}_{s}\right)\right|^{2} d s\right\}\right]^{\frac{1}{8 q_{\varepsilon}}} \exp \left\{2\left(8 q_{\varepsilon}-1\right) \int_{0}^{T}|\phi(s)|^{2} d s\right\} .
\end{aligned}
$$

Altogether, we obtain

$$
\left|S\left(Y_{t}^{i, b_{n}}-Y_{t}^{i, b}\right)(\phi)\right| \leq \mathrm{const} \cdot E\left[J_{n}\right]^{\frac{1}{1+\varepsilon}} \cdot \exp \left\{2\left(8 \frac{1+\varepsilon}{\varepsilon}-1\right) \int_{0}^{T}|\phi(s)|^{2} d s\right\} .
$$

Lemma 2.12. Let $b_{n}:[0, T] \times \mathbb{R}^{d} \longrightarrow \mathbb{R}^{d}$ be a sequence of smooth functions with compact support which approximates the coefficient $b:[0, T] \times \mathbb{R}^{d} \longrightarrow \mathbb{R}^{d}$ in $L_{p}^{q}$. Then for any $0 \leq t \leq T$ there exists a subsequence of the corresponding strong solutions $X_{n_{j}, t}=Y_{t}^{b_{n_{j}}}, j=1,2, \ldots$, such that

$$
Y_{t}^{b_{n_{j}}} \longrightarrow Y_{t}^{b}
$$

for $j \rightarrow \infty$ in $L^{2}(\Omega)$. In particular this implies $Y_{t}^{b} \in L^{2}(\Omega), 0 \leq t \leq T$.

Proof. By Corollary 2.9 we know that there exists a subsequence $Y_{t}^{b_{n_{j}}}, j \geq 1$, converging in $L^{2}(\Omega)$. Further, we need to show that $E\left[J_{n_{j}}\right] \rightarrow 0$ as $j \rightarrow \infty$ with $J_{n_{j}}$ as in (32). To this end, observe that for a function $f \in L_{p}^{q}$ one has

$$
E\left[\int_{0}^{T} f\left(s, \tilde{B}_{s}\right) d s\right]=\int_{0}^{T}(2 \pi s)^{-d / 2} \int_{\mathbb{R}^{d}} f(s, z) e^{-|z|^{2} /(2 s)} d z d s .
$$

Then by using Hölder's inequality with respect to $z$ and then to $s$ we see that for any $p^{\prime}, q^{\prime} \in[1, \infty]$ satisfying

$$
\frac{d}{p^{\prime}}+\frac{2}{q^{\prime}}<2
$$

we have

$$
E\left[\int_{0}^{T} f\left(s, \tilde{B}_{s}\right) d s\right] \leq C\|f\|_{L_{p^{\prime}}^{q^{\prime}}}
$$

where $C$ is a constant depending on $T, d, p^{\prime}, q^{\prime}$. Then from condition (7), since $p, q>2$ we can find an $\delta \in[0,1)$ small enough so that $p, q>2(1+\delta)$. For these $p, q$ define $p^{\prime}:=\frac{p}{2(1+\delta)} \geq 1$ and $q:=\frac{q^{\prime}}{2(1+\delta)}>1$ and apply the above estimate to $|f|^{2(1+\delta)}$ to obtain

$$
E\left[\int_{0}^{T}\left|f\left(s, \tilde{B}_{s}\right)\right|^{2(1+\delta)} d s\right] \leq C\|f\|_{L_{p}^{q}}
$$

Now since $b_{n}^{(j)}-b^{(j)} \in L_{p}^{q}$ for every $j=1, \ldots, d$ and $0<\frac{1+\varepsilon}{2}<1$ we have

$$
E\left[\left(\int_{0}^{T}\left(b_{n}^{(j)}\left(s, \tilde{B}_{s}^{(j)}\right)-b^{(j)}\left(s, \tilde{B}_{s}^{(j)}\right)\right)^{2} d s\right)^{\frac{1+\varepsilon}{2}}\right] \leq E\left[\int_{0}^{T}\left(b_{n}^{(j)}\left(s, \tilde{B}_{s}^{(j)}\right)-b^{(j)}\left(s, \tilde{B}_{s}^{(j)}\right)\right)^{2} d s\right]^{\frac{1+\varepsilon}{2}}
$$


which goes to zero by the above estimate (34) by just taking the case where $\delta=0$.

Finally, for the second term in $E\left[J_{n_{j}}\right]$ we have

$$
\begin{aligned}
E & {\left[\left|\int_{0}^{T}\left(b^{(j)}\left(s, \tilde{B}_{s}^{(j)}\right)^{2}-b_{n}^{(j)}\left(s, \tilde{B}_{s}^{(j)}\right)^{2}\right) d s\right|^{1+\varepsilon}\right] } \\
& \leq T^{\varepsilon} E\left[\int_{0}^{T}\left(b^{(j)}\left(s, \tilde{B}_{s}^{(j)}\right)+b_{n}^{(j)}\left(s, \tilde{B}_{s}^{(j)}\right)\right)^{1+\varepsilon}\left(b^{(j)}\left(s, \tilde{B}_{s}^{(j)}\right)-b_{n}^{(j)}\left(s, \tilde{B}_{s}^{(j)}\right)\right)^{1+\varepsilon} d s\right] \\
& \leq T^{\varepsilon} \int_{0}^{T} E\left[\left(b^{(j)}\left(s, \tilde{B}_{s}^{(j)}\right)+b_{n}^{(j)}\left(s, \tilde{B}_{s}^{(j)}\right)\right)^{2(1+\varepsilon)}\right]^{1 / 2} E\left[\left(b^{(j)}\left(s, \tilde{B}_{s}^{(j)}\right)-b_{n}^{(j)}\left(s, \tilde{B}_{s}^{(j)}\right)\right)^{2(1+\varepsilon)}\right]^{1 / 2} d s \\
& \leq T^{\varepsilon} E\left[\int_{0}^{T}\left(b^{(j)}\left(s, \tilde{B}_{s}^{(j)}\right)+b_{n}^{(j)}\left(s, \tilde{B}_{s}^{(j)}\right)\right)^{2(1+\varepsilon)} d s\right]^{1 / 2} E\left[\int_{0}^{T}\left(b^{(j)}\left(s, \tilde{B}_{s}^{(j)}\right)-b_{n}^{(j)}\left(s, \tilde{B}_{s}^{(j)}\right)\right)^{2(1+\varepsilon)} d s\right]^{1 / 2} .
\end{aligned}
$$

Then since $b^{(j)}+b_{n}(j) \in L_{p}^{q}$ for every $n \geq 0$ we have

$$
\sup _{n \geq 0} E\left[\int_{0}^{T}\left(b^{(j)}\left(s, \tilde{B}_{s}^{(j)}\right)+b_{n}^{(j)}\left(s, \tilde{B}_{s}^{(j)}\right)\right)^{2(1+\varepsilon)} d s\right]^{1 / 2}<\infty
$$

for a sufficiently small $\varepsilon \in(0,1)$ by Lemma B.2 and

$$
E\left[\int_{0}^{T}\left(b^{(j)}\left(s, \tilde{B}_{s}^{(j)}\right)-b_{n}^{(j)}\left(s, \tilde{B}_{s}^{(j)}\right)\right)^{2(1+\varepsilon)} d s\right]^{1 / 2} \rightarrow 0
$$

as $n \rightarrow \infty$ by estimate (34) for a sufficiently small $\varepsilon>0$.

Thus, by Lemma 2.11, $Y_{t}^{b_{n_{j}}} \rightarrow Y_{t}^{b}$ as $j \rightarrow \infty$ in $(\mathcal{S})^{*}$. But then, by uniqueness of the limit, also $Y_{t}^{b_{n_{j}}} \rightarrow Y_{t}^{b}$ in $L^{2}(\Omega)$.

Remark 2.13. It follows from the above proof that $Y_{t}^{b_{n}} \rightarrow Y_{t}^{b}$ as $n \rightarrow \infty$ in $L^{2}\left(\Omega ; \mathbb{R}^{d}\right)$ for all $t$ and $x$.

In fact, Lemma 2.12 enables us now to state the following "transformation property" for $Y_{t}^{b}$ which is essentially a consequence of the $L^{2}(\Omega)$ convergence of the approximating solutions.

This transformation property serves as a tool to directly verify that the limiting process is a strong solution. Uniqueness then follows by Lemma B.2 and with these two results we conclude the last two steps of our programme.

Lemma 2.14. Assume that $b:[0, T] \times \mathbb{R}^{d} \longrightarrow \mathbb{R}^{d}$ is in $L_{p}^{q}$. Then

$$
\varphi^{(i)}\left(t, Y_{t}^{b}\right)=E_{\widetilde{\mu}}\left[\varphi^{(i)}\left(t, \widetilde{B}_{t}\right) \mathcal{E}_{T}^{\diamond}(b)\right]
$$

a.e. for all $0 \leq t \leq T, i=1, \ldots, d$ and $\varphi=\left(\varphi^{(1)}, \ldots, \varphi^{(d)}\right)$ such that $\varphi\left(t, B_{t}\right) \in L^{2}\left(\Omega ; \mathbb{R}^{d}\right)$.

Proof. See [33, Lemma 16] or [26].

Using the above auxiliary results we can finally give the proof of Theorem 2.1.

Proof of Theorem 2.1. We want to use the transformation property (35) of Lemma 2.14 to show that $Y_{t}^{b}$ is a unique strong solution of the $\operatorname{SDE}(5)$. To shorten notation we set $\int_{0}^{t} \varphi(s, \omega) d B_{s}:=\sum_{j=1}^{d} \int_{0}^{t} \varphi^{(j)}(s, \omega) d B_{s}^{(j)}$ and $x=0$. Also, let $b_{n}, n=0,1, \ldots$, be a sequence of functions as required in Lemma 2.12 .

We comment on that $Y^{b}$ has a continuous modification. The latter can be seen as follows: Since each $Y_{t}^{b_{n}}$ is a strong solution of the SDE (5) with respect to the drift $b_{n}$ we obtain from Girsanov's theorem and our assumptions that

$$
E_{\mu}\left[\left(Y_{t}^{i, b_{n}}-Y_{u}^{i, b_{n}}\right)^{4}\right]=E_{\widetilde{\mu}}\left[\left(\widetilde{B}_{t}^{(i)}-\widetilde{B}_{u}^{(i)}\right)^{4} \mathcal{E}\left(\int_{0}^{T} b_{n}\left(s, \widetilde{B}_{s}\right) d \widetilde{B}_{s}\right)\right] \leq \mathrm{const} \cdot|t-u|^{2}
$$


for all $0 \leq u, t \leq T, n \geq 0, i=1, \ldots, d$. The above constant comes from the fact that $\left\{\mathcal{E}\left(\int_{0}^{T} b_{n}\left(s, \widetilde{B}_{s}\right) d \widetilde{B}_{s}\right)\right\}_{n \geq 0}$ is bounded in $L^{2}\left(\Omega ; \mathbb{R}^{d}\right)$ with respect to the measure $\tilde{\mu}$, see Lemma 3.2. in [20] or Lemma B.1.

By Remark 2.13 we know that

$$
Y_{t}^{b_{n}} \longrightarrow Y_{t}^{b} \text { in } L^{2}\left(\Omega ; \mathbb{R}^{d}\right)
$$

and hence we have almost sure convergence for a further subsequence, $0 \leq t \leq T$. So we get that by Fatou's lemma

$$
E_{\mu}\left[\left(Y_{t}^{i, b}-Y_{u}^{i, b}\right)^{4}\right] \leq \mathrm{const} \cdot|t-u|^{2}
$$

for all $0 \leq u, t \leq T, i=1, \ldots, d$. Then Kolmogorov's lemma guarantees a continuous modification of $Y_{t}^{b}$.

Since $\widetilde{B}_{t}$ is a weak solution of (5) for the drift $b(s, x)+\phi(s)$ with respect to the measure $d \mu^{*}=\mathcal{E}\left(\int_{0}^{T}\left(b\left(s, \widetilde{B}_{s}\right)+\right.\right.$ $\left.\phi(s)) d \widetilde{B}_{s}\right) d \mu$ we get that

$$
\begin{aligned}
S\left(Y_{t}^{i, b}\right)(\phi) & =E_{\widetilde{\mu}}\left[\widetilde{B}_{t}^{(i)} \mathcal{E}\left(\int_{0}^{T}\left(b\left(s, \widetilde{B}_{s}\right)+\phi(s)\right) d \widetilde{B}_{s}\right)\right] \\
& =E_{\mu^{*}}\left[\widetilde{B}_{t}^{(i)}\right] \\
& =E_{\mu^{*}}\left[\int_{0}^{t}\left(b^{(i)}\left(s, \widetilde{B}_{s}\right)+\phi^{(i)}(s)\right) d s\right] \\
& =\int_{0}^{t} E_{\widetilde{\mu}}\left[b^{(i)}\left(s, \widetilde{B}_{s}\right) \mathcal{E}\left(\int_{0}^{T}\left(b\left(u, \widetilde{B}_{u}\right)+\phi(u)\right) d \widetilde{B}_{u}\right)\right] d s+S\left(B_{t}^{(i)}\right)(\phi) .
\end{aligned}
$$

Thus the transformation property (35) applied to $b$ yields

$$
S\left(Y_{t}^{i, b}\right)(\phi)=S\left(\int_{0}^{t} b^{(i)}\left(u, Y_{u}^{i, b}\right) d u\right)(\phi)+S\left(B_{t}^{(i)}\right)(\phi) .
$$

Then it follows from the injectivity of the $S$-transform that

$$
Y_{t}^{b}=\int_{0}^{t} b\left(s, Y_{s}^{b}\right) d s+B_{t}
$$

See Section A in the Appendix.

The Malliavin differentiability of $Y_{t}^{b}$ comes from the fact that $Y_{t}^{i, b_{n}} \rightarrow Y_{t}^{i, b}$ in $L^{2}(\Omega)$ and

$$
\sup _{n \geq 0}\left\|Y_{t}^{i, b_{n}}\right\|_{\mathbb{D}^{1,2}} \leq M<\infty
$$

for all $i=1, \ldots, d$ and $0 \leq t \leq T$. See e.g. [30].

On the other hand, using uniqueness in law, which is a consequence of Lemma B.2 and Proposition 3.10, Ch. 5 in [18] we may apply, under our conditions, Girsanov's theorem to any other solution. Then the proof of Proposition 2.4 (see e.g. [32, Proposition 1]) shows that any other solution necessarily coincides with $Y_{t}^{b}$.

We conclude this section with a generalisation of Theorem 2.1 to a class of non-degenerate $d$-dimensional Itôdiffusions.

Theorem 2.15. Assume the time-homogeneous $\mathbb{R}^{d}$-valued SDE

$$
d X_{t}=b\left(X_{t}\right) d t+\sigma\left(X_{t}\right) d B_{t}, \quad X_{0}=x \in \mathbb{R}^{d}, 0 \leq t \leq T,
$$

where the coefficients $b: \mathbb{R}^{d} \longrightarrow \mathbb{R}^{d}$ and $\sigma: \mathbb{R}^{d} \longrightarrow \mathbb{R}^{d} \times \mathbb{R}^{d}$ are Borel measurable. Suppose that there exists a bijection $\Lambda: \mathbb{R}^{d} \longrightarrow \mathbb{R}^{d}$, which is twice continuously differentiable. Let $\Lambda_{x}: \mathbb{R}^{d} \longrightarrow L\left(\mathbb{R}^{d}, \mathbb{R}^{d}\right)$ and $\Lambda_{x x}: \mathbb{R}^{d} \longrightarrow$ 
$L\left(\mathbb{R}^{d} \times \mathbb{R}^{d}, \mathbb{R}^{d}\right)$ be the corresponding derivatives of $\Lambda$ and assume that

$$
\Lambda_{x}(y) \sigma(y)=\mathrm{id}_{\mathbb{R}^{d}} \quad \text { for } y \text { a.e. }
$$

as well as

$$
\Lambda^{-1} \text { is Lipschitz continuous. }
$$

Require that the function $b_{*}: \mathbb{R}^{d} \longrightarrow \mathbb{R}^{d}$ given by

$$
\begin{aligned}
b_{*}(x):= & \Lambda_{x}\left(\Lambda^{-1}(x)\right)\left[b\left(\Lambda^{-1}(x)\right)\right] \\
& +\frac{1}{2} \Lambda_{x x}\left(\Lambda^{-1}(x)\right)\left[\sum_{i=1}^{d} \sigma\left(\Lambda^{-1}(x)\right)\left[e_{i}\right], \sum_{i=1}^{d} \sigma\left(\Lambda^{-1}(x)\right)\left[e_{i}\right]\right]
\end{aligned}
$$

satisfies the conditions of Theorem 2.1 , where $e_{i}, i=1, \ldots, d$, is a basis of $\mathbb{R}^{d}$. Then there exists a Malliavin differentiable solution $X_{t}$ to (37).

Proof. The proof can be directly obtained from Itô's Lemma. See [27].

\section{Application to the Bismut-Elworthy-Li formula}

As an application we want to use Theorem 2.1 to derive a Bismut-Elworthy-Li formula for solutions $v$ to the Kolmogorov equation

$$
\frac{\partial}{\partial t} v(t, x)=\sum_{j=1}^{d} b_{j}(t, x) \frac{\partial}{\partial x_{j}} v(t, x)+\frac{1}{2} \sum_{i=1}^{d} \frac{\partial^{2}}{\partial x_{i}^{2}} v(t, x)
$$

with initial condition $v(0, x)=\Phi(x)$, where $b:[0, T] \times \mathbb{R}^{d} \rightarrow \mathbb{R}^{d}$ belongs to $L_{p}^{q}$.

It is known that, see [20] or [10], that when $\Phi$ is continuous and bounded there exists a solution to (38) given by

$$
v(t, x)=E\left[\Phi\left(X_{t}^{x}\right)\right],
$$

where $v$ is a solution to the Kolmogorov Equation (38) which is unique among all bounded solutions in the space $H_{2, p}^{q}$, as introduced in Theorem 2.2, with $p, q>2$ satisfying (7). Moreover, $\frac{\partial}{\partial x} v \in L^{\infty}\left([0, T] \times \mathbb{R}^{d}\right)$.

In the sequel, we aim at finding a representation for $\frac{\partial}{\partial x} v$ without using derivatives of $\Phi$. See Theorem 4.6 in [25] in the case of $b \in L^{\infty}\left([0, T] \times \mathbb{R}^{d}\right)$.

Theorem 3.1 (Bismut-Elworthy-Li formula). Assume $\Phi \in C_{b}\left(\mathbb{R}^{d}\right)$ and let $U$ be an open, bounded subset of $\mathbb{R}^{d}$. Then the derivative of the solution to (38) can be represented as

$$
\frac{\partial}{\partial x} v(t, x)=E\left[\Phi\left(X_{t}^{x}\right) \int_{0}^{t} a(s)\left(\frac{\partial}{\partial x} X_{s}^{x}\right)^{*} d B_{s}\right]^{*}
$$

for almost all $x \in U$ and all $t \in(0, T]$, where $a$ is any bounded measurable function such that $\int_{0}^{t} a(s) d s=1$ and where $*$ denotes the transposition of matrices.

Proof. The proof is similar to Theorem 4.6 in [25] in the case of $b \in L^{\infty}\left([0, T] \times \mathbb{R}^{d}\right)$. For the convenience of the reader we give the full proof.

Assume that $\Phi \in C_{b}^{2}\left(\mathbb{R}^{d}\right)$ (the general case of $\Phi \in C_{b}\left(\mathbb{R}^{d}\right)$ can be proved by approximation of $\Phi$ in relation (42)) and let $b_{n}$ and $X_{t}^{n, x}$ be as in the previous section. If we replace $b$ by $b_{n}$ in (38) we have the unique solution 
given by

$$
v_{n}(t, x)=E\left[\Phi\left(X_{t}^{n, x}\right)\right] .
$$

By using Remark 2.13 we see that $v_{n}(t, x) \rightarrow v(t, x)$ for each $t$ and $x$.

By [30, Page 109] we have that

$$
D_{s} X_{t}^{n, x} \frac{\partial}{\partial x} X_{s}^{n, x}=\frac{\partial}{\partial x} X_{t}^{n, x}, \quad 0<s \leq t \leq T,
$$

where the above product is the usual matrix product. So it follows that

$$
\frac{\partial}{\partial x} X_{t}^{n, x}=\int_{0}^{t} a(s) D_{s} X_{t}^{n, x} \frac{\partial}{\partial x} X_{s}^{n, x} d s .
$$

Interchanging integration and differentiation in connection with the chain rule we find that

$$
\begin{aligned}
\frac{\partial}{\partial x} v_{n}(t, x) & =E\left[\Phi^{\prime}\left(X_{t}^{n, x}\right) \frac{\partial}{\partial x} X_{t}^{n, x}\right] \\
& =E\left[\int_{0}^{t} a(s) D_{s} \Phi\left(X_{t}^{n, x}\right) \frac{\partial}{\partial x} X_{s}^{n, x} d s\right] \\
& =E\left[\Phi\left(X_{t}^{n, x}\right) \int_{0}^{t} a(s)\left(\frac{\partial}{\partial x} X_{s}^{n, x}\right)^{*} d B_{s}\right]^{*},
\end{aligned}
$$

where we applied the chain rule and the duality formula for the Malliavin derivative to the last equality.

Choose $\varphi \in C_{0}^{\infty}(U)$. In what follows, we will prove that

$$
\int_{\mathbb{R}^{d}} \frac{\partial}{\partial x} \varphi(x) v(t, x) d x=-\int_{\mathbb{R}^{d}} \varphi(x) E\left[\Phi\left(X_{t}^{x}\right) \int_{0}^{t} a(s)\left(\frac{\partial}{\partial x} X_{s}^{x}\right)^{*} d B_{s}\right]^{*} d x .
$$

In fact, dominated convergence combined with Remark 2.13 gives

$$
\begin{aligned}
\int_{\mathbb{R}^{d}} \frac{\partial}{\partial x} \varphi(x) v(t, x) d x= & -\lim _{n \rightarrow \infty} \int_{\mathbb{R}^{d}} \varphi(x) E\left[\Phi\left(X_{t}^{n, x}\right) \int_{0}^{t} a(s)\left(\frac{\partial}{\partial x} X_{s}^{n, x}\right)^{*} d B_{s}\right]^{*} d x \\
= & -\lim _{n \rightarrow \infty} \int_{\mathbb{R}^{d}} \varphi(x) E\left[\left(\Phi\left(X_{t}^{n, x}\right)-\Phi\left(X_{t}^{x}\right)\right) \int_{0}^{t} a(s)\left(\frac{\partial}{\partial x} X_{s}^{n, x}\right)^{*} d B_{s}\right]^{*} d x \\
& -\lim _{n \rightarrow \infty} \int_{\mathbb{R}^{d}} \varphi(x) E\left[\Phi\left(X_{t}^{x}\right) \int_{0}^{t} a(s)\left(\frac{\partial}{\partial x} X_{s}^{n, x}\right)^{*} d B_{s}\right]^{*} d x \\
= & -\lim _{n \rightarrow \infty}(i)_{n}-\lim _{n \rightarrow \infty}(i i)_{n} .
\end{aligned}
$$

As for the first term we get

$$
(i)_{n} \leq \int_{\mathbb{R}^{d}}|\varphi(x)|\left\|\frac{\partial}{\partial x} \Phi\right\|_{\infty}\left\|X_{t}^{n, x}-X_{t}^{x}\right\|_{L^{2}\left(\Omega ; \mathbb{R}^{d}\right)}\|a\|_{\infty}\left(\sup _{k \geq 1, s \in[0, T]} E\left[\left\|\frac{\partial}{\partial x} X_{s}^{k, x}\right\|_{\mathbb{R}^{d \times d}}^{2}\right]\right)^{1 / 2} d x,
$$

which goes to zero as $n$ tends to infinity by Lebesque dominated convergence theorem, Remark 2.13 and Remark 2.8.

For the second term, (ii) $)_{n}$ since $X_{t}^{x}$ is Malliavin differentiable and $\Phi \in C_{b}^{2}\left(\mathbb{R}^{d}\right)$ it follows from the Clark-Ocone formula that (see e.g. [30])

$$
\Phi\left(X_{t}^{x}\right)=E\left[\Phi\left(X_{t}^{x}\right)\right]+\int_{0}^{t} E\left[D_{s} \Phi\left(X_{t}^{x}\right) \mid \mathcal{F}_{s}\right] d B_{s}
$$


So

$$
\begin{aligned}
(\text { ii })_{n} & =\int_{\mathbb{R}^{d}} \varphi(x) E\left[\Phi\left(X_{t}^{x}\right) \int_{0}^{t} a(s)\left(\frac{\partial}{\partial x} X_{s}^{n, x}\right)^{*} d B_{s}\right]^{*} d x \\
& =\int_{\mathbb{R}^{d}} \varphi(x) E\left[\left(E\left[\Phi\left(X_{t}^{x}\right)\right]+\int_{0}^{t} E\left[D_{s} \Phi\left(X_{t}^{x}\right) \mid \mathcal{F}_{s}\right] d B_{s}\right) \int_{0}^{t} a(s)\left(\frac{\partial}{\partial x} X_{s}^{n, x}\right)^{*} d B_{s}\right]^{*} d x \\
& =\int_{0}^{t} a(s) \int_{\mathbb{R}^{d}} \varphi(x) E\left[D_{s} \Phi\left(X_{t}^{x}\right) \frac{\partial}{\partial x} X_{s}^{n, x}\right] d x d s .
\end{aligned}
$$

One checks by means of Theorem 2.6 that $\varphi(\cdot) D_{s} \Phi\left(X_{i}\right)=\varphi(\cdot) \Phi^{\prime}\left(X_{i}\right) D_{s} X_{i}$ belongs to $L^{2}\left(\mathbb{R}^{d} \times \Omega ; \mathbb{R}^{d}\right)$ so that for each $s$, the function

$$
g_{n}(s)=\int_{\mathbb{R}^{d}} \varphi(x) E\left[D_{s} \Phi\left(X_{t}^{x}\right) \frac{\partial}{\partial x} X_{s}^{n, x}\right] d x
$$

converges to $\int_{\mathbb{R}^{d}} \varphi(x) E\left[D_{s} \Phi\left(X_{t}^{x}\right) \frac{\partial}{\partial x} X_{s}^{x}\right] d x$ by the weak convergence of $\frac{\partial}{\partial x} X_{s}^{n, x}$ in $L^{2}([0, T] \times U \times \Omega)$ for a subsequence in virtue of Remark 2.8. Further,

$$
\begin{aligned}
\left|g_{n}(s)\right| & \leq \int_{\mathbb{R}^{d}}|\varphi(x)|\left\|D_{s} \Phi\left(X_{t}^{x}\right)\right\|_{L^{2}\left(\Omega ; \mathbb{R}^{d}\right)}\left\|\frac{\partial}{\partial x} X_{s}^{n, x}\right\|_{L^{2}\left(\Omega ; \mathbb{R}^{d}\right)} d x \\
& \leq \sup _{y \in \mathbb{R}^{d}, u \leq t, k \in \mathbb{N}}\left\|D_{u} \Phi\left(X_{t}^{y}\right)\right\|_{L^{2}\left(\Omega ; \mathbb{R}^{d}\right)}\left\|\frac{\partial}{\partial x} X_{u}^{k, y}\right\|_{L^{2}\left(\Omega ; \mathbb{R}^{d}\right)} \int_{\mathbb{R}^{d}}|\varphi(x)| d x
\end{aligned}
$$

so that Lebesgue's dominated convergence theorem gives

$$
\lim _{n \rightarrow \infty}(i i)_{n}=\int_{0}^{t} a(s) \int_{\mathbb{R}^{d}} \varphi(x) E\left[D_{s} \Phi\left(X_{t}^{x}\right) \frac{\partial}{\partial x} X_{s}^{x}\right] d x d s .
$$

By reversing equations (43), (44) and (45) with $\frac{\partial}{\partial x} X_{s}^{x}$ in place of $\frac{\partial}{\partial x} X_{s}^{n, x}$ we obtain the result.

\section{Appendix A: Framework}

In this appendix we collect some facts from Gaussian white noise analysis and Malliavin calculus, which we shall use in Section 2 to construct strong solutions of SDE's. See [15,21,31] for more information on white noise theory. As for Malliavin calculus the reader may consult $[5,23,24,30]$.

\section{A.1. Basic facts of Gaussian white noise theory}

A crucial step in our proof for the constuction of strong solutions (see Section 3) relies on a generalised stochastic process in the Hida distribution space which is shown to be a SDE solution. Let us first recall the definition of this space which is due to T. Hida (see [15]).

From now on we fix a time horizon $0<T<\infty$. Let $A$ be a (positive) self-adjoint operator on $L^{2}([0, T])$ with $\operatorname{Spec}(A)>1$. Require that $A^{-r}$ is of Hilbert-Schmidt type for some $r>0$ and let $\left\{e_{j}\right\}_{j \geq 0}$ be a complete orthonormal basis of $L^{2}([0, T])$ in $\operatorname{Dom}(A)$ and let $\lambda_{j}>0, j \geq 0$ be the eigenvalues of $A$ such that

$$
1<\lambda_{0} \leq \lambda_{1} \leq \cdots \rightarrow \infty \text {. }
$$

Suppose that each basis element $e_{j}$ is a continuous function on $[0, T]$. Further let $O_{\lambda}, \lambda \in \Gamma$, be an open covering of $[0, T]$ such that

$$
\sup _{j \geq 0} \lambda_{j}^{-\alpha(\lambda)} \sup _{t \in O_{\lambda}}\left|e_{j}(t)\right|<\infty
$$


for $\alpha(\lambda) \geq 0$.

In the sequel let $\mathcal{S}([0, T])$ be the standard countably Hilbertian space constructed from $\left(L^{2}([0, T]), A\right)$. See [31]. Then $\mathcal{S}([0, T])$ is a nuclear subspace of $L^{2}([0, T])$. The topological dual of $\mathcal{S}([0, T])$ is denoted by $\mathcal{S}^{\prime}([0, T])$. Then the Bochner-Minlos theorem entails the existence of a unique probability measure $\pi$ on $\mathcal{B}\left(\mathcal{S}^{\prime}([0, T])\right.$ ) (Borel $\sigma$-algebra of $\left.\mathcal{S}^{\prime}([0, T])\right)$ such that

$$
\int_{\mathcal{S}^{\prime}([0, T])} e^{i\langle\omega, \phi\rangle} \pi(d \omega)=e^{-\frac{1}{2}\|\phi\|_{L^{2}([0, T])}^{2}}
$$

for all $\phi \in \mathcal{S}([0, T])$, where $\langle\omega, \phi\rangle$ stands for the action of $\omega \in \mathcal{S}^{\prime}([0, T])$ on $\phi \in \mathcal{S}([0, T])$. Define

$$
\Omega_{i}=\mathcal{S}^{\prime}([0, T]), \quad \mathcal{F}_{i}=\mathcal{B}\left(\mathcal{S}^{\prime}([0, T])\right), \quad \mu_{i}=\pi,
$$

for $i=1, \ldots, d$. Then the product measure

$$
\mu=\underset{i=1}{\stackrel{X}{X}} \mu_{i}
$$

on the measurable space

$$
(\Omega, \mathcal{F}):=\left(\prod_{i=1}^{d} \Omega_{i}, \bigotimes_{i=1}^{d} \mathcal{F}_{i}\right)
$$

is called $d$-dimensional white noise probability measure.

Consider the Doléans-Dade exponential

$$
\widetilde{e}(\phi, \omega)=\exp \left(\langle\omega, \phi\rangle-\frac{1}{2}\|\phi\|_{L^{2}\left([0, T] ; \mathbb{R}^{d}\right)}^{2}\right),
$$

for $\omega=\left(\omega_{1}, \ldots, \omega_{d}\right) \in\left(\mathcal{S}^{\prime}([0, T])\right)^{d}$ and $\phi=\left(\phi^{(1)}, \ldots, \phi^{(d)}\right) \in(\mathcal{S}([0, T]))^{d}$, where $\langle\omega, \phi\rangle:=\sum_{i=1}^{d}\left\langle\omega_{i}, \phi_{i}\right\rangle$.

Now let $\left((\mathcal{S}([0, T]))^{d}\right)^{\widehat{\otimes} n}$ be the $n$th completed symmetric tensor product of $(\mathcal{S}([0, T]))^{d}$ with itself. One checks that $\widetilde{e}(\phi, \omega)$ is holomorphic in $\phi$ around zero. Hence, there exist generalised Hermite polynomials $H_{n}(\omega) \in$ $\left(\left((\mathcal{S}([0, T]))^{d}\right)^{\widehat{\otimes} n}\right)^{\prime}$ such that

$$
\widetilde{e}(\phi, \omega)=\sum_{n \geq 0} \frac{1}{n !}\left\langle H_{n}(\omega), \phi^{\otimes n}\right\rangle
$$

for $\phi$ in a certain neighbourhood of zero in $(\mathcal{S}([0, T]))^{d}$. One proves that

$$
\left\{\left\langle H_{n}(\omega), \phi^{(n)}\right\rangle: \phi^{(n)} \in\left((\mathcal{S}([0, T]))^{d}\right)^{\widehat{\otimes} n}, n \in \mathbb{N}_{0}\right\}
$$

is a total set of $L^{2}(\Omega)$. Further, it can be shown that the generalised Hermite polynomials satisfy the orthogonality relation

$$
\int_{\mathcal{S}^{\prime}}\left\langle H_{n}(\omega), \phi^{(n)}\right\rangle\left\langle H_{m}(\omega), \psi^{(m)}\right\rangle \mu(d \omega)=\delta_{n, m} n !\left(\phi^{(n)}, \psi^{(n)}\right)_{L^{2}\left([0, T]^{n} ;\left(\mathbb{R}^{d}\right)^{\otimes n}\right)}
$$

for all $n, m \in \mathbb{N}_{0}, \phi^{(n)} \in\left((\mathcal{S}([0, T]))^{d}\right)^{\widehat{\otimes} n}, \psi^{(m)} \in\left((\mathcal{S}([0, T]))^{d}\right)^{\widehat{\otimes} m}$ where

$$
\delta_{n, m}= \begin{cases}1 & \text { if } n=m \\ 0 & \text { else }\end{cases}
$$


Denote by $\widehat{L}^{2}\left([0, T]^{n} ;\left(\mathbb{R}^{d}\right)^{\otimes n}\right)$ the space of square integrable symmetric functions $f\left(x_{1}, \ldots, x_{n}\right)$ with values in $\left(\mathbb{R}^{d}\right)^{\otimes n}$. Then it follows from relation (50) that the mappings

$$
\phi^{(n)} \longmapsto\left\langle H_{n}(\omega), \phi^{(n)}\right\rangle
$$

from $\left(\mathcal{S}([0, T])^{d}\right)^{\widehat{\otimes} n}$ to $L^{2}(\Omega)$ have unique continuous extensions

$$
I_{n}: \widehat{L}^{2}\left([0, T]^{n} ;\left(\mathbb{R}^{d}\right)^{\otimes n}\right) \longrightarrow L^{2}(\Omega)
$$

for all $n \in \mathbb{N}$. These extensions $I_{n}\left(\phi^{(n)}\right)$ can be identified as $n$-fold iterated Itô integrals of $\phi^{(n)} \in \widehat{L}^{2}\left([0, T]^{n} ;\left(\mathbb{R}^{d}\right)^{\otimes n}\right)$ with respect to a $d$-dimensional Wiener process

$$
B_{t}=\left(B_{t}^{(1)}, \ldots, B_{t}^{(d)}\right)
$$

on the white noise space

$$
(\Omega, \mathcal{F}, \mu)
$$

We mention that square integrable functionals of $B_{t}$ admit a Wiener-Itô chaos representation which can be regarded as an infinite-dimensional Taylor expansion, that is

$$
L^{2}(\Omega)=\bigoplus_{n \geq 0} I_{n}\left(\widehat{L}^{2}\left([0, T]^{n} ;\left(\mathbb{R}^{d}\right)^{\otimes n}\right)\right) .
$$

The definition of the Hida stochastic test function and distribution space is based on the Wiener-Itô chaos decomposition (53): Set

$$
A^{d}:=(A, \ldots, A)
$$

Using a second quantisation argument, the Hida stochastic test function space $(\mathcal{S})$ is defined as the space of all $f=\sum_{n \geq 0}\left\langle H_{n}(\cdot), \phi^{(n)}\right\rangle \in L^{2}(\Omega)$ such that

$$
\|f\|_{0, p}^{2}:=\sum_{n \geq 0} n !\left\|\left(\left(A^{d}\right)^{\otimes n}\right)^{p} \phi^{(n)}\right\|_{L^{2}\left([0, T]^{n} ;\left(\mathbb{R}^{d}\right)^{\otimes n}\right)}^{2}<\infty
$$

for all $p \geq 0$. In fact, the space $(\mathcal{S})$ is a nuclear Fréchet algebra with respect to multiplication of functions and its topology is induced by the seminorms $\|\cdot\|_{0, p}, p \geq 0$. Further one shows that

$$
\widetilde{e}(\phi, \omega) \in(\mathcal{S})
$$

for all $\phi \in(\mathcal{S}([0, T]))^{d}$.

On the other hand, the topological dual of $(\mathcal{S})$, denoted by $(\mathcal{S})^{*}$, is called Hida stochastic distribution space. Using these definitions we ontain the Gel'fand triple

$$
(\mathcal{S}) \hookrightarrow L^{2}(\Omega) \hookrightarrow(\mathcal{S})^{*} .
$$

It turns out that the white noise of the coordinates of the $d$-dimensional Wiener process $B_{t}$, that is the time derivatives

$$
W_{t}^{i}:=\frac{d}{d t} B_{t}^{i}, \quad i=1, \ldots, d,
$$

belong to $(\mathcal{S})^{*}$. 
We also recall the definition of the $S$-transform. See [32]. The $S$-transform of a $\Phi \in(\mathcal{S})^{*}$, denoted by $S(\Phi)$, is defined by the dual pairing

$$
S(\Phi)(\phi)=\langle\Phi, \widetilde{e}(\phi, \omega)\rangle
$$

for $\phi \in\left(\mathcal{S}_{\mathbb{C}}([0, T])\right)^{d}$. Here $\mathcal{S}_{\mathbb{C}}([0, T])$ the complexification of $\mathcal{S}([0, T])$. The $S$-transform is a monomorphism from $(\mathcal{S})^{*}$ to $\mathbb{C}$. In particular, if

$$
S(\Phi)=S(\Psi) \quad \text { for } \Phi, \Psi \in(\mathcal{S})^{*}
$$

then

$$
\Phi=\Psi .
$$

As an example one finds that

$$
S\left(W_{t}^{i}\right)(\phi)=\phi^{i}(t), \quad i=1, \ldots, d
$$

for $\phi=\left(\phi^{(1)}, \ldots, \phi^{(d)}\right) \in\left(\mathcal{S}_{\mathbb{C}}([0, T])\right)^{d}$.

Finally, we recall the concept of the Wick or Wick-Grassmann product. The Wick product defines a tensor algebra multiplication on the Fock space and is introduced as follows: The Wick product of two distributions $\Phi, \Psi \in(\mathcal{S})^{*}$, denoted by $\Phi \diamond \Psi$, is the unique element in $(\mathcal{S})^{*}$ such that

$$
S(\Phi \diamond \Psi)(\phi)=S(\Phi)(\phi) S(\Psi)(\phi)
$$

for all $\phi \in\left(\mathcal{S}_{\mathbb{C}}([0, T])\right)^{d}$. As an example, we get

$$
\left\langle H_{n}(\omega), \phi^{(n)}\right\rangle \diamond\left\langle H_{m}(\omega), \psi^{(m)}\right\rangle=\left\langle H_{n+m}(\omega), \phi^{(n)} \widehat{\otimes} \psi^{(m)}\right\rangle
$$

for $\phi^{(n)} \in\left((\mathcal{S}([0, T]))^{d}\right)^{\widehat{\otimes} n}$ and $\psi^{(m)} \in\left((\mathcal{S}([0, T]))^{d}\right)^{\widehat{\otimes} m}$. The latter in connection with (48) implies that

$$
\widetilde{e}(\phi, \omega)=\exp ^{\diamond}(\langle\omega, \phi\rangle)
$$

for $\phi \in(\mathcal{S}([0, T]))^{d}$. Here the Wick exponential $\exp ^{\diamond}(X)$ of a $X \in(\mathcal{S})^{*}$ is defined as

$$
\exp ^{\diamond}(X)=\sum_{n \geq 0} \frac{1}{n !} X^{\diamond n},
$$

where $X^{\diamond n}=X \diamond \cdots \diamond X$, provided that the sum on the right hand side converges in $(\mathcal{S})^{*}$.

\section{A.2. Basic elements of Malliavin calculus}

In this section we pass in review some basic definitions from Malliavin calculus.

For convenience we consider the case $d=1$. Let $F \in L^{2}(\Omega)$. Then we know from (53) that

$$
F=\sum_{n \geq 0}\left\langle H_{n}(\cdot), \phi^{(n)}\right\rangle
$$

for unique $\phi^{(n)} \in \widehat{L}^{2}\left([0, T]^{n}\right)$. Suppose that

$$
\sum_{n \geq 1} n n !\left\|\phi^{(n)}\right\|_{L^{2}\left([0, T]^{n}\right)}^{2}<\infty
$$


Then the Malliavin derivative $D_{t}$ of $F$ in the direction of $B_{t}$ can be defined as

$$
D_{t} F=\sum_{n \geq 1} n\left\langle H_{n-1}(\cdot), \phi^{(n)}(\cdot, t)\right\rangle .
$$

We denote by $\mathbb{D}^{1,2}$ the space of all $F \in L^{2}(\Omega)$ such that (65) holds. The Malliavin derivative $D$. is a linear operator from $\mathbb{D}^{1,2}$ to $L^{2}([0, T] \times \Omega)$. We mention that $\mathbb{D}^{1,2}$ is a Hilbert space with the norm $\|\cdot\|_{1,2}$ given by

$$
\|F\|_{1,2}^{2}:=\|F\|_{L^{2}(\Omega, \mu)}^{2}+\|D \cdot F\|_{L^{2}([0, T] \times \Omega, \lambda \times \mu)}^{2} .
$$

We get the following chain of continuous inclusions:

$$
(\mathcal{S}) \hookrightarrow \mathbb{D}^{1,2} \hookrightarrow L^{2}(\Omega) \hookrightarrow \mathbb{D}^{-1,2} \hookrightarrow(\mathcal{S})^{*},
$$

where $\mathbb{D}^{-1,2}$ is the dual of $\mathbb{D}^{1,2}$.

\section{Appendix B: Technical results}

We give a list of technical results needed for the proofs of Sections 2 and 3.

Lemma B.1. Let $\left\{f_{n}\right\}_{n \geq 0}$ be a bounded sequence of functions in $L_{p}^{q}$. Then, for every $k \in \mathbb{R}$

$$
\sup _{x \in \mathbb{R}^{d}} \sup _{n \geq 0} E\left[\exp \left\{k \int_{0}^{T}\left|f_{n}\left(s, x+B_{s}\right)\right|^{2} d s\right\}\right]<\infty .
$$

In particular, there exists a weak solution to SDE (5).

Proof. See [20, Lemma 3.2].

Lemma B.2. Let $\left\{f_{n}\right\}_{n \geq 0}$ a sequence of elements in $L^{p, q}$ that converges to some $f \in L^{p, q}$. Then there exists $\varepsilon>1$ such that

$$
\sup _{n \geq 0} E\left[\int_{0}^{T}\left\|f_{n}\left(s, \phi_{s}^{n}\right)\right\|^{2 \varepsilon} d s\right]<\infty .
$$

Here $\phi_{s}^{n}: x \mapsto X_{t}^{x, n}$ denotes the stochastic flow associated to the solution of the SDE (5) with drift coefficient $b_{n} \in$ $\mathcal{C}_{b}^{\infty}\left(\mathbb{R}^{d}\right)$.

Proof. See [9, Lemma 15].

We also need the following crucial lemma, which can be found in [8, Lemma 3.4].

Lemma B.3. Let $U_{n}$ be the solution of the PDE (10) with $\Phi=b=b_{n} \in \mathcal{C}_{b}^{\infty}\left(\mathbb{R}^{n}\right)$. Let $X_{t}^{x, n}$ be the solution of the SDE (5) with drift coefficient $b_{n} \in \mathcal{C}_{b}^{\infty}\left(\mathbb{R}^{d}\right)$. Then the following holds true

(i) For each $r>0$ there exists a function $f$ with $\lim _{n} f(n)=0$ such that

$$
\sup _{x \in B_{r}} \sup _{t \in[0, T]}\left\|U_{n}(t, x)-U(t, x)\right\| \leq f(n)
$$

and

$$
\sup _{x \in B_{r}} \sup _{t \in[0, T]}\left\|\nabla U_{n}(t, x)-\nabla U(t, x)\right\| \leq f(n) .
$$


(ii) There exists a $\lambda \in \mathbb{R}$ for which $\sup _{t \in[0, T]}\left\|\nabla U_{n}(t, x)\right\| \leq \frac{1}{2}$.

(iii) $\sup _{n \geq 0}\left\|\Delta U_{n}(t, x)\right\|_{L^{p, q}}<\infty$.

(iv) As a consequence of the boundedness of $U_{n}$ and $\nabla U_{n}$ we have

$$
\sup _{t \in[0, T]} E\left[\left\|\gamma_{t}^{n}(x)\right\|^{a}\right] \leq C\left(1+|x|^{a}\right) .
$$

The following lemma gives a bound for the derivative of the inverse of the family of diffeomorphisms $\gamma_{t}$. See [8, Lemma 3.5] for its proof.

Lemma B.4. Let $\gamma_{t, n}: \mathbb{R}^{d} \rightarrow \mathbb{R}^{d}$ be the $C^{1}$-diffeomorphisms defined as $\gamma_{t, n}(x):=x+U_{n}(t, x)$ for $x \in \mathbb{R}^{d}$ associated to $X_{t}^{x, n}$ the solution of $S D E(5)$ with drift coefficient $b_{n} \in C_{b}^{\infty}\left(\mathbb{R}^{d}\right)$. Then

$$
\sup _{n \geq 0} \sup _{t \in[0, T]}\left\|\nabla \gamma_{t, n}^{-1}\right\|_{C\left(\mathbb{R}^{d}\right)} \leq 2 .
$$

The next result was shown in [7, Corollary 13].

Lemma B.5. Let $V_{t}^{n}$ be the process defined in (22). Then for every $\alpha \in \mathbb{R}$

$$
\sup _{n \geq 0} E\left[e^{\alpha V_{T}^{n}}\right] \leq C
$$

Observe that the same estimate holds for any $t \in[0, T]$ since $V_{t}^{n}$ is an increasing process.

\section{Appendix C}

The following result which is due to [4, Theorem 1] gives a compactness criterion for subsets of $L^{2}\left(\Omega ; \mathbb{R}^{d}\right)$ using Malliavin calculus.

Theorem C.1. Let $\{(\Omega, \mathcal{A}, P) ; H\}$ be a Gaussian probability space, that is $(\Omega, \mathcal{A}, P)$ is a probability space and $H$ a separable closed subspace of Gaussian random variables of $L^{2}(\Omega)$, which generate the $\sigma$-field $\mathcal{A}$. Denote by $\mathbf{D}$ the derivative operator acting on elementary smooth random variables in the sense that

$$
\mathbf{D}\left(f\left(h_{1}, \ldots, h_{n}\right)\right)=\sum_{i=1}^{n} \partial_{i} f\left(h_{1}, \ldots, h_{n}\right) h_{i}, \quad h_{i} \in H, f \in C_{b}^{\infty}\left(\mathbb{R}^{n}\right) .
$$

Further let $\mathbf{D}_{1,2}$ be the closure of the family of elementary smooth random variables with respect to the norm

$$
\|F\|_{1,2}:=\|F\|_{L^{2}(\Omega)}+\|\mathbf{D} F\|_{L^{2}(\Omega ; H)} .
$$

Assume that $C$ is a self-adjoint compact operator on $H$ with dense image. Then for any $c>0$ the set

$$
\mathcal{G}=\left\{G \in \mathbf{D}_{1,2}:\|G\|_{L^{2}(\Omega)}+\left\|C^{-1} \mathbf{D} G\right\|_{L^{2}(\Omega ; H)} \leq c\right\}
$$

is relatively compact in $L^{2}(\Omega)$.

A useful bound in connection with Theorem C.1, based on fractional Sobolev spaces is the following (see [4]):

Lemma C.2. Let $v_{s}, s \geq 0$ be the Haar basis of $L^{2}([0, T])$. For any $0<\alpha<1 / 2$ define the operator $A_{\alpha}$ on $L^{2}([0, T])$ by

$$
A_{\alpha} v_{s}=2^{k \alpha} v_{s}, \quad \text { if } s=2^{k}+j
$$


for $k \geq 0,0 \leq j \leq 2^{k}$ and

$$
A_{\alpha} T=T .
$$

Then for all $\beta$ with $\alpha<\beta<(1 / 2)$, there exists a constant $c_{1}$ such that

$$
\left\|A_{\alpha} f\right\| \leq c_{1}\left\{\|f\|_{L^{2}([0, T])}+\left(\int_{0}^{T} \int_{0}^{T} \frac{\left|f(t)-f\left(t^{\prime}\right)\right|^{2}}{\left|t-t^{\prime}\right|^{1+2 \beta}} d t d t^{\prime}\right)^{1 / 2}\right\} .
$$

A direct consequence of Theorem C. 1 and Lemma C.2 is now the following compactness criterion which is essential for the proof of Corollary 2.9.

Corollary C.3. Let a sequence of $\mathcal{F}_{T}$-measurable random variables $X_{n} \in \mathbb{D}_{1,2}, n=1,2, \ldots$, be such that there exist constants $\alpha>0$ and $C>0$ with

$$
\begin{gathered}
\sup _{n} E\left[\left|X_{n}\right|^{2}\right] \leq C, \\
\sup _{n} E\left[\left\|D_{t} X_{n}-D_{t^{\prime}} X_{n}\right\|^{2}\right] \leq C\left|t-t^{\prime}\right|^{\alpha} \\
\text { for } 0 \leq t^{\prime} \leq t \leq T \text { and } \\
\sup _{n} \sup _{0 \leq t \leq T} E\left[\left\|D_{t} X_{n}\right\|^{2}\right] \leq C .
\end{gathered}
$$

Then the sequence $X_{n}, n=1,2, \ldots$, is relatively compact in $L^{2}(\Omega)$.

\section{References}

[1] D. R. Baños, T. Nilssen and F. Proske. Strong existence and higher order Fréchet differentiability of stochastic flows of fractional Brownian motion driven SDE's with singular drift. Available at arXiv:1511.02717.

[2] A. S. Chernyi. On strong and weak uniqueness for stochastic differential equations. Teor. Veroyatn. Primen. 46 (3) (2001) $483-497$.

[3] G. Da Prato, F. Flandoli, E. Priola and M. Röckner. Strong uniqueness for stochastic evolution equations in Hilbert spaces perturbed by a bounded measurable drift. Ann. Probab. 41 (5) (2013) 3306-3344.

[4] G. Da Prato, P. Malliavin and D. Nualart. Compact families of Wiener functionals. C. R. Math. Acad. Sci. Paris 315 (Série I) (1992) 12871291.

[5] G. Di Nunno, B. Øksendal and F. Proske. Malliavin Calculus for Lévy Processes with Applications to Finance. Springer, Berlin, 2008.

[6] H. J. Engelbert. On the theorem of T. Yamada and S. Watanabe. Stoch. Stoch. Rep. 36 (3-4) (1991) 205-216. MR1128494

[7] E. Fedrizzi and F. Flandoli. Pathwise uniqueness and continuous dependence for SDE's with non-regular drift. Stochastics 83 (3) (2011) 241-257.

[8] E. Fedrizzi and F. Flandoli. Hölder flow and differentiability for SDEs with nonregular drift. Stoch. Anal. Appl. 31 (4) (2013) $708-736$.

[9] E. Fedrizzi and F. Flandoli. Noise prevents singularities in linear transport equations. J. Funct. Anal. 264 (6) (2013) $1329-1354$.

[10] F. Flandoli. Random Pertubation of PDEs and Fluid Dynamic Models. École d'Été de Probabilitiés de Saint-Flour XL-2010. Lecture Notes in Mathematics. Springer, 2011.

[11] F. Flandoli, M. Gubinelli and E. Priola. Well-posedness of the transport equation by stochastic perturbation. Invent. Math. 180 (1) (2010) 1-53. MR2593276

[12] F. Flandoli, T. Nilssen and F. Proske. Malliavin differentiability and strong solutions for a class of SDE in Hilbert spaces. Preprint, 2015.

[13] I. Gyöngy and N. V. Krylov. Existence of strong solutions for Itô's stochastic equations via approximations. Probab. Theory Related Fields 105 (1996) 143-158.

[14] S. Haadem and F. Proske. On the construction and Malliavin differentiability of solutions of Lévy noise driven SDE's with singular coefficients. J. Funct. Anal. 266 (8) (2014) 5321-5359.

[15] T. Hida, H.-H. Kuo, J. Potthoff and L. Streit. White Noise: An Infinite-Dimensional Calculus. Kluwer Academic Publishers, 1993.

[16] J. Jacod. Weak and strong solutions of stochastic differential equations. Stochastics 3 (3) (1980) 171-191. MR0573202

[17] G. Kallianpur and J. Xiong. Stochastic Differential Equations in Infinite-Dimensional Spaces. Institute of Mathematical Statistics, Hayward, CA, 1995.

[18] I. Karatzas and S. E. Shreve. Brownian Motion and Stochastic Calculus, 2nd edition. Springer-Verlag, New York, 1991.

[19] N. V. Krylov. Some properties of traces for stochastic and deterministic parabolic weighted Sobolev spaces. J. Funct. Anal. 183 (1) (2001) $1-41$. 
[20] N. V. Krylov and M. Röckner. Strong solutions of stochastic equations with singular time dependent drift. Probab. Theory Related Fields 131 (2) (2005) 154-196.

[21] H.-H. Kuo. White Noise Distribution Theory. Soch. Series, CRC Press, Boca Raton, FL, 1996.

[22] A. Lanconelli and F. Proske. On explicit strong solutions of Itô-SDE's and the Donsker delta function of a diffusion. Anal. Quantum Probab. Relat. Top. 7 (3) (2004).

[23] P. Malliavin. Stochastic calculus of variations and hypoelliptic operators. In Proc. Inter. Symp. on Stoch. Diff. Equations (Kyoto, 1976) 195263. Wiley, 1978. MR0536013

[24] P. Malliavin. Stochastic Analysis. Grundlehren der Mathematischen Wissenschaften. Springer, Berlin, 1997.

[25] O. Menoukeu-Pamen, T. Meyer-Brandis, T. Nilssen, F. Proske and T. Zhang. A variational approach to the construction and Malliavin differentiability of strong solutions of SDE's. Math. Ann. 357 (2) (2013) 761-799. MR3096525

[26] T. Meyer-Brandis and F. Proske. On the existence and explicit representability of strong solutions of Lévy noise driven SDE's with irregular coefficients. Communications in Mathematical Sciences 4 (1) (2006).

[27] T. Meyer-Brandis and F. Proske. Construction of strong solutions of SDE's via Malliavin calculus. J. Funct. Anal. 258 (2010) $3922-3953$.

[28] S.-E. A. Mohammed, T. Nilssen and F. Proske. Sobolev differentiable stochastic flows of SDE's with measurable drift and applications. Ann. Probab. 43 (3) (2015) 1535-1576.

[29] T. Nilssen. One-dimensional SDE's with discontinuous, unbounded drift and continuously differentiable solutions to the stochastic transport equation. Preprint. MR3484042

[30] D. Nualart. The Malliavin Calculus and Related Topics, 2nd edition. Springer-Verlag, Berlin, 2006.

[31] N. Obata. White Noise Calculus and Fock Space. LNM 1577, Springer-Verlag, Berlin, 1994.

[32] J. Potthoff and L. Streit. A characterization of Hida distributions. J. Funct. Anal. 101 (1991) 212-229.

[33] F. Proske. Stochastic differential equations - some new ideas. Stochastics 79 (2007) 563-600. MR2368369

[34] A. Y. Veretennikov. On the strong solutions of stochastic differential equations. Theory Probab. Appl. 24 (1979) $354-366$.

[35] T. Yamada and S. Watanabe. On the uniqueness of solutions of stochastic differential equations. J. Math. Kyoto Univ. II (1971) $155-167$.

[36] A. K. Zvonkin. A transformation of the state space of a diffusion process that removes the drift. Math. USSR, Sb. 22 (1974) 129-149. 